Hartree-Fock Theory with a Self-Generated Magnetic Field

\title{
Hartree-Fock Theory with a Self-Generated Magnetic Field
}

S. Comelli, a) and C.J. García-Cervera ${ }^{1,2, b)}$

1) Mathematics Department, University of California, Santa Barbara, CA 93106, USA.

2) Visiting Professor at BCAM - Basque Center for Applied Mathematics, Mazarredo 14, E48009 Bilbao, Basque Country - Spain

(Dated: 23 May 2017)

We prove existence of a ground state within the Hartree-Fock theory for atoms and molecules, in the presence of self-generated magnetic fields, with and without direct spin coupling. The ground state exists provided that the total charge $Z$ of the $K$ nuclei exceeds $N$, where $N$ is the number of electrons, and, in the spin-polarized case, provided in addition that the nuclear charge is not too high.

a) Electronic mail: scomelli@math.ucsb.edu.

b) Electronic mail: cgarcia@math.ucsb.edu. 
Hartree-Fock Theory with a Self-Generated Magnetic Field

\section{INTRODUCTION}

In this paper we study the Hartree-Fock model for a system of electrons interacting with static nuclei through the Coulomb potential, in the presence of direct coupling. In particular, we prove existence of a ground state when the system is neutral or positively charged. We consider a system of $N$ electrons and $K$ nuclei of charge $\left(Z_{1}, \ldots, Z_{K}\right), Z_{k}>0$ for all $k$, in the Born-Oppenheimer approximation. The many-body Hamiltonian including electron spin-magnetic field interactions that describes such a system system is:

$$
H_{\mathrm{SM}}=\sum_{j=1}^{N} \frac{1}{2}\left[\sigma_{j} \cdot\left(i \nabla_{j}-\mathbf{A}\left(\mathbf{x}_{j}\right)\right)\right]^{2}+\left(v\left(\mathbf{x}_{j}\right)+\sum_{1 \leq i<j \leq N} \frac{1}{\left|\mathbf{x}_{i}-\mathbf{x}_{j}\right|}\right) \mathbb{I}_{2 \times 2},
$$

where

$$
v(\mathbf{x})=-\sum_{k=1}^{K} \frac{Z_{k}}{\left|\mathbf{x}-\mathbf{R}_{k}\right|}
$$

is the potential generated by the nuclei, $\mathbf{R}_{k}$ is the position of the $k$-th nucleus and $\mathbb{I}_{2 \times 2}$ is the $2 \times 2$ identity matrix. The Hamiltonian $H_{\mathrm{SM}}$ acts on the Hilbert space of normalized, $\mathrm{N}$ particle, antisymmetric electron wave functions defined by

$$
\mathcal{H}=\left\{\Psi \in \bigwedge_{j=1}^{N} L^{2}\left(\mathbb{R}^{3}, \mathbb{C}^{2}\right):\|\Psi\|_{L^{2}\left(\mathbb{R}^{3 N}, \mathbb{C}^{2 N}\right)}=1\right\}
$$

In other words, the electron wave function $\Psi\left(\mathbf{x}_{1}, s_{1}, \ldots, \mathbf{x}_{N}, s_{N}\right) \in \bigwedge_{j=1}^{N} L^{2}\left(\mathbb{R}^{3}, \mathbb{C}^{2}\right)$ is a function of both spatial and spin variables, $\mathbf{x}_{j} \in \mathbb{R}^{3}$ and $s_{j} \in\left\{-\frac{1}{2}, \frac{1}{2}\right\}$. As a consequence of the Pauli Exclusion Principle, $\Psi$ is antisymmetric in the indexes labeling the particles. In addition, $\Psi$ is normalized in the following sense:

$$
\|\Psi\|_{L^{2}\left(\mathbb{R}^{3 N}, \mathbb{C}^{2^{N}}\right)}^{2}=\sum_{s_{1}, \ldots, s_{N}} \int_{\mathbb{R}^{3}} \cdots \int_{\mathbb{R}^{3}}\left|\Psi\left(\mathbf{x}_{1}, s_{1}, \ldots, \mathbf{x}_{N}, s_{N}\right)\right|^{2} d \mathbf{x}_{1} \cdots d \mathbf{x}_{N}=1 .
$$

The inner product on $\mathcal{H}$ is defined as:

$$
(\Psi, \Phi)_{L^{2}\left(\mathbb{R}^{3 N}, \mathbb{C}^{2 N}\right)}=\sum_{s_{1}, \ldots, s_{N}} \int_{\mathbb{R}^{3}} \cdots \int_{\mathbb{R}^{3}} \Psi\left(\mathbf{x}_{1}, s_{1}, \ldots, \mathbf{x}_{N}, s_{N}\right) \bar{\Phi}\left(\mathbf{x}_{1}, s_{1}, \ldots, \mathbf{x}_{N}, s_{N}\right) d \mathbf{x}_{1} \cdots d \mathbf{x}_{N}
$$

The form domain of $H_{\mathrm{SM}}$, which corresponds to the set of admissible wave functions, is:

$$
\mathcal{W}=\left\{\Psi \in \bigwedge_{j=1}^{N} L^{2}\left(\mathbb{R}^{3}, \mathbb{C}^{2}\right):\|\Psi\|_{L^{2}\left(\mathbb{R}^{3 N}, \mathbb{C}^{2 N}\right)}=1,\|\nabla \Psi\|_{L^{2}\left(\mathbb{R}^{3 N}, \mathbb{C}^{2^{N}}\right)}<+\infty\right\}
$$


The first term in (1) is the Pauli kinetic energy operator, and $\mathbf{A}$ is the magnetic vector potential. In this paper we assume that there are no externally applied magnetic fields, and therefore the magnetic potential appearing in (1) is the one self-generated by the orbital motion of the electrons.

The Pauli kinetic energy operator $\sigma_{j}$ acts on the j-th variables of $\Psi \in \mathcal{W}$ and contains the Pauli matrices:

$$
\sigma_{j}=\left(\sigma_{j x}, \sigma_{j y}, \sigma_{j z}\right)=\left(\left(\begin{array}{ll}
0 & 1 \\
1 & 0
\end{array}\right),\left(\begin{array}{cc}
0 & -i \\
i & 0
\end{array}\right),\left(\begin{array}{cc}
1 & 0 \\
0 & -1
\end{array}\right)\right) .
$$

The action of this operator on the electron wave function can be easily understood if we think of the electron wave function in terms of spinor: in other words, $\Psi$ is regarded as a $2^{N}$-components vector valued function of the space variables. Each component corresponds to a possible configuration of values for the $N$ spin variables (the electron spin has only two possible values: $-1 / 2$ or $1 / 2$ ). Clearly, if we are dealing with an operator that acts on one variable only (such as the Pauli operator), then, in a similar way, we will treat $\Psi$ as a two components vector valued function, one for each possible value of the spin of the variable involved. In the case of the one-electron wave function, for example, $\psi \in L^{2}\left(\mathbb{R}^{3}, \mathbb{C}^{2}\right)$ is a two component vector defined as:

$$
\psi(\mathbf{x})=\left(\begin{array}{c}
\psi^{+}(\mathbf{x}) \\
\psi^{-}(\mathbf{x})
\end{array}\right)=\left(\begin{array}{c}
\psi(\mathbf{x}, 1 / 2) \\
\psi(\mathbf{x},-1 / 2)
\end{array}\right) .
$$

The non-relativistic quantum energy of the system is given by:

$$
\begin{aligned}
\mathcal{E}_{\mathrm{SM}}(\Psi, \mathbf{A}) & =\frac{1}{2} \sum_{j=1}^{N} \int_{\mathbb{R}^{3 N}}\left|\sigma_{j} \cdot\left(i \nabla_{j}-\mathbf{A}\left(\mathbf{x}_{j}\right)\right) \Psi\right|_{\mathbb{C}^{2}}^{2} \\
& +\left(\Psi,\left(\sum_{j=1}^{N} v\left(\mathbf{x}_{j}\right)+\sum_{1 \leq i<j \leq N} \frac{1}{\left|\mathbf{x}_{i}-\mathbf{x}_{j}\right|}\right) \Psi\right)_{L^{2}\left(\mathbb{R}^{3 N}, \mathbb{C}^{2 N}\right)}+\frac{1}{\alpha^{2}} \int_{\mathbb{R}^{3}}|\nabla \times \mathbf{A}|^{2} .
\end{aligned}
$$

Note that $\mathbf{B}=\nabla \times \mathbf{A}$ is the magnetic field generated by $\mathbf{A}$, and therefore the last term in (2) is the magnetic field energy. The Hamiltonians (1) and (2) are dimensionless in the Hartree units. In particular, in these units, $\alpha=\frac{e^{2}}{4 \pi \epsilon_{0} \hbar c} \approx \frac{1}{137.04}$ is the fine structure constant, where $\hbar$ is the Planck's constant divided by $2 \pi, e$ is the elementary charge, $c$ is the speed of light and $\epsilon_{0}$ is the permittivity of vacuum. 
If we include the interactions between the electron's self-generated magnetic field (that is, the magnetic field due to the electron's orbital motion) and the intrinsic electron spin into our model, then we must define the ground state energy of the system as:

$$
E_{\mathrm{SM}}=\inf \left\{\mathcal{E}_{\mathrm{SM}}(\Psi, \mathbf{A}):(\Psi, \mathbf{A}) \in \mathcal{D}_{\mathrm{SM}}\right\}
$$

where

$$
\mathcal{D}_{\mathrm{SM}}=\left\{(\Psi, \mathbf{A}): \begin{array}{l}
\Psi \in \mathcal{W} \\
\quad \mathbf{A} \in L^{6}\left(\mathbb{R}^{3}\right) ; \nabla \cdot \mathbf{A}=0 ; \nabla \times \mathbf{A} \in L^{2}\left(\mathbb{R}^{3}\right)
\end{array}\right\}
$$

Note that the minimization in (3) is carried among both the wave functions and the magnetic vector potential. For the vector potential A, we choose the Coulomb gauge, i.e. we assume that $\nabla \cdot \mathbf{A}=0$.

Problem (3) was studied in a series of papers by Fröhlich, Lieb, Loss, Yau and Solovej (see Refs. 1, 2, 3 and 4). In Ref. 1, Fröhlich, Lieb and Loss proved that, in the case of the one-electron atom $(N=1, K=1)$, there is a critical nuclear charge, $Z_{c}$, for which the atom is stable (i.e. its ground state energy is finite) for $Z<Z_{c}$, and unstable for $Z>Z_{c}$ (Z being the charge of the atom). When the atom is stable, the authors also proved existence of a ground state wave function. In Ref. 2, Lieb and Loss considered the problem of stability for the many-electrons atom ( $N$ arbitrary, $K=1)$ and the one-electron molecule $(N=1, K$ arbitrary), under some restrictions on the nuclear charges as well. Finally, in Ref. 3, Loss and Yau showed that the Pauli operator $\sigma \cdot(i \nabla-\mathbf{A})$ has zero as an eigenvalue. This means that there exist non zero solutions to $\sigma \cdot(i \nabla-\mathbf{A}) \Psi=0$. As a consequence of this, the kinetic energy might not be enough to control the potential and magnetic energies. Specifically, if we rescale $\Psi$, we can drive the total energy to $-\infty$ for certain values of the nuclear charge and obtain instability. This is not possible when direct coupling is not present, as a consequence of the Lieb-Thirring and Diamagnetic inequalities (see Ref. 5 and Ref. 6, respectively) which provide the bound:

$$
\left\|\rho_{\Psi}\right\|_{L^{5 / 3}\left(\mathbb{R}^{3}\right)}^{5 / 3} \leq\|(i \nabla-\mathbf{A}) \Psi\|_{L^{2}\left(\mathbb{R}^{3 N}, \mathbb{C}^{2 N}\right)}^{2},
$$

where $\rho_{\Psi}$ is the total electronic density associated with $\Psi$, that is

$$
\rho_{\Psi}(\mathbf{x})=N \sum_{\tau \in\left\{-\frac{1}{2}, \frac{1}{2}\right\}^{N-1}} \int_{\mathbb{R}^{3(N-1)}}|\Psi(\mathbf{x}, \mathbf{y}, \tau)|_{\mathbb{C}^{2}}^{2} \mathrm{~d} \mathbf{y}
$$


The problem of stability for the many-electrons many-nuclei system was finally solved by Lieb, Loss and Solovej in Ref. 4. The authors proved stability under suitable values of the nuclear charge (the range of admissible charges was later extended in Ref. 7). They also prove a Lieb-Thirring type of inequality for the Pauli operator. A generalization of the same inequality is showed in Ref. 8 by Bugliaro, Fefferman, Fröhlich, Graf and Stubbe. In that case, the stability of matter with magnetic fields follows as a by-product of it.

In this paper, we focus on proving the existence of a ground state in the Hartree-Fock theory with direct coupling and self-generated field. A Hartree-Fock theory starts by considering wave functions of the following form:

$$
\Psi\left(\mathbf{x}_{1}, s_{1}, \ldots, \mathbf{x}_{N}, s_{N}\right)=\frac{1}{\sqrt{N !}} \operatorname{det}\left(\psi_{k}\left(\mathbf{x}_{j}, s_{j}\right)\right)=\frac{1}{\sqrt{N !}}\left|\begin{array}{ccc}
\psi_{1}\left(\mathbf{x}_{1}, s_{1}\right) & \cdots & \psi_{1}\left(\mathbf{x}_{N}, s_{N}\right) \\
\vdots & & \vdots \\
\psi_{N}\left(\mathbf{x}_{1}, s_{1}\right) & \cdots & \psi_{N}\left(\mathbf{x}_{N}, s_{N}\right)
\end{array}\right|,
$$

where $\psi_{1}, \ldots, \psi_{N}$ is an orthonormal set of one-electron wave functions, that is $\psi_{j} \in$ $H^{1}\left(\mathbb{R}^{3}, \mathbb{C}^{2}\right)$ for $j=1, \ldots, N$ and $\left(\psi_{j}, \psi_{k}\right)_{L^{2}\left(\mathbb{R}^{3}, \mathbb{C}^{2}\right)}=\delta_{j k}$.

We denote by $\mathcal{W}_{\text {slater }}$ the subset of $\mathcal{W}$ consisting of all finite energy Slater determinants. Standard algebraic calculations show that the quantum energy (2) for a Slater determinant $\Psi\left(\mathbf{x}_{1}, s_{1}, \ldots, \mathbf{x}_{N}, s_{N}\right)=\frac{1}{\sqrt{N !}} \operatorname{det}\left(\psi_{k}\left(\mathbf{x}_{j}, s_{j}\right)\right)$ is given by

$$
\begin{aligned}
\mathcal{E}_{\mathrm{SMHF}}\left(\psi_{1}, \cdots, \psi_{N}, \mathbf{A}\right) & =\frac{1}{2} \sum_{j=1}^{N} \int_{\mathbb{R}^{3}}\left|\sigma \cdot(i \nabla-\mathbf{A}) \psi_{j}\right|_{\mathbb{C}^{2}}^{2}+\int_{\mathbb{R}^{3}} \rho v \\
& +\frac{1}{2} \iint_{\mathbb{R}^{3} \times \mathbb{R}^{3}} \frac{\rho(\mathbf{x}) \rho(\mathbf{y})-\operatorname{Tr}_{\mathbb{C}^{2}}\left(|\gamma(\mathbf{x}, \mathbf{y})|^{2}\right)}{|\mathbf{x}-\mathbf{y}|} d \mathbf{x} d \mathbf{y}+\frac{1}{\alpha^{2}} \int_{\mathbb{R}^{3}}|\nabla \times \mathbf{A}|^{2}
\end{aligned}
$$

where $\rho(\mathbf{x})=\sum_{j=1}^{N}\left|\psi_{j}(\mathbf{x})\right|_{\mathbb{C}^{2}}^{2}$ is the total electron density associated with the Slater determinant and $\gamma(\mathbf{x}, \mathbf{y})=\sum_{j=1}^{N} \psi_{j}(\mathbf{x}) \cdot \overline{\psi_{j}}(\mathbf{y}) \in_{2 \times 2}(\mathbb{C})$, is a $2 \times 2$ matrix.

We adopt here the same notations as in Ref. 3: for vector fields ( $\mathbf{A}$ or $\mathbf{B}$ )

$$
\|\mathbf{A}\|_{L^{2}}=\left\|(\mathbf{A} \cdot \mathbf{A})^{1 / 2}\right\|_{L^{2}\left(\mathbb{R}^{3}\right)},
$$

where $\mathbf{A}=\left(A_{1}, A_{2}, A_{3}\right)$ and $\mathbf{A} \cdot \mathbf{A}=\sum_{j}\left|A_{j}\right|^{2}$. For spinors $\psi$

$$
\|\psi\|_{L^{2}}=\left\|(\psi \cdot \psi)^{1 / 2}\right\|_{L^{2}\left(\mathbb{R}^{3}\right)},
$$


where $(\psi \cdot \psi)(\mathbf{x})=|\psi(\mathbf{x})|_{\mathbb{C}^{2}}^{2}=\left|\psi^{+}(\mathbf{x})\right|^{2}+\left|\psi^{-}(\mathbf{x})\right|^{2}$. Finally, for gradients $\nabla \mathbf{A}$ or $\nabla \psi$,

$$
\|\nabla \mathbf{A}\|_{L^{2}}=\|\nabla \mathbf{A}\|_{L^{2}\left(\mathbb{R}^{3}, \mathbb{R}^{3}\right)}=\left\|\left(\sum_{j, k=1}^{3}\left|\partial_{j} A_{k}\right|^{2}\right)^{1 / 2}\right\|_{L^{2}\left(\mathbb{R}^{3}\right)}=\left\{\sum_{j, k=1}^{3} \int_{\mathbb{R}^{3}}\left|\partial_{j} A_{k}\right|^{2}\right\}^{1 / 2}
$$

and, analogously,

$$
\begin{aligned}
\|\nabla \psi\|_{L^{2}}=\|\nabla \psi\|_{L^{2}\left(\mathbb{R}^{3}, \mathbb{C}^{2}\right)} & =\left\|\left(\sum_{j=1}^{3}\left(\left|\partial_{j} \psi^{+}\right|^{2}+\left|\partial_{j} \psi^{-}\right|^{2}\right)\right)^{1 / 2}\right\|_{L^{2}\left(\mathbb{R}^{3}\right)} \\
& =\left\{\sum_{j=1}^{3} \int_{\mathbb{R}^{3}}\left(\left|\partial_{j} \psi^{+}\right|^{2}+\left|\partial_{j} \psi^{-}\right|^{2}\right)\right\}^{1 / 2} .
\end{aligned}
$$

In the absence of magnetic fields, the existence of a Hartree-Fock ground state for Coulomb systems was proved by Lieb and Simon (see Ref. 9) and Lions (see Ref. 10), under the condition $N \leq Z$. A different proof, combining non linear and geometric techniques, has been developed by Friesecke in Ref. 11 and Lewin in Ref. 12 .

In Ref. 13, Enstedt and Melgaard extended this result to the case in which a fixed magnetic field is applied (that is they minimize the energy (4) in the wave functions only). However, the authors do not include the spin-field interactions, and make some strong assumptions on the vector potential (e.g. they assumed it to be homogeneous of degree -1). An alternative solution to the same problem is presented by Esteban and Lions in Ref. 14: they consider a larger class of vector potentials (i.e. $\mathbf{A} \in L_{\text {loc }}^{3}\left(\mathbb{R}^{3}\right)$ ), but do not include the spin in their model and the field is not determined self-consistently. The condition $N \leq Z$ ensures that the total charge of the nuclei should be sufficiently positive to prevent the electrons from escaping to infinity. It is a sufficient condition, but whether it is necessary is an open question. Results in this direction were presented by Lieb in Ref. 15, where he proved that for $N \geq 2 Z+K$ there is no ground state for the spinless magnetic Hartree-Fock for bounded vector potentials. This bound was improved in the absence of magnetic fields by Solovej in Ref. 16, who proved that there exists a universal constant $Q>0$ such that for all positive integers satisfying $N \geq Z+Q$ there are no minimizers for the Hartree-Fock functional.

Finally, recall that, when no magnetic field is present, a fundamental result by Lieb and $\mathrm{Si}-$ mon (see Ref. 17) implies that Hartree-Fock theory is asymptotically exact for large nuclear charges and electron numbers, in the sense that, in the limit for $N$ and $Z$ going to infinity, the Hartree-Fock theory provides the leading terms in the ground state energy. As it was 
established by Erdós and Solovej in Ref. 18, the inclusion of a self-generated magnetic field does not change the asymptotics of the leading term: in fact, the effect of the magnetic field appears only in the Scott term.

In the context of Kohn-Sham density-functional theory (see Ref. 19), Gontier proved existence of a ground state in Ref. 20 in the presence of a fixed external magnetic field, extending earlier work by Anantharaman and Cancès on the Kohn-Sham equations to the magnetic case (see Ref. 21).

In our work, we consider spin-orbit interactions and prove existence of a ground state for a wider class of vector potentials, namely $\mathbf{A} \in L^{6}\left(\mathbb{R}^{3}\right)$ and $\nabla \times \mathbf{A} \in L^{2}\left(\mathbb{R}^{3}\right)$, which are self-generated. We define the Hartree-Fock ground state energy as:

$$
E_{\mathrm{SMHF}}=\inf \left\{\mathcal{E}_{\mathrm{SMHF}}\left(\psi_{1}, \ldots, \psi_{N}, \mathbf{A}\right):\left(\psi_{1}, \ldots, \psi_{N}, \mathbf{A}\right) \in \mathcal{D}_{\mathrm{SMHF}}\right\}
$$

where

$$
\mathcal{D}_{\mathrm{SMHF}}=\left\{\left(\psi_{1}, \ldots, \psi_{N}, \mathbf{A}\right): \begin{array}{l}
\psi_{j} \in H^{1}\left(\mathbb{R}^{3}, \mathbb{C}^{2}\right), j=1, \ldots, N ;\left(\psi_{j}, \psi_{k}\right)_{L^{2}\left(\mathbb{R}^{3}, \mathbb{C}^{2}\right)}=\delta_{j, k} \\
\mathbf{A} \in L^{6}\left(\mathbb{R}^{3}\right) ; \nabla \cdot \mathbf{A}=0 ; \nabla \times \mathbf{A} \in L^{2}\left(\mathbb{R}^{3}\right)
\end{array}\right\} .
$$

Any minimizer to (6) is called a Hartree-Fock ground state.

Although the classical formulation of the Hartree-Fock problem involves the wave functions, from a mathematical point of view, it is more convenient to work with density matrices. This approach, originally proposed by Gilbert (see Ref. 22) and subsequently generalized by Valone (see Ref. 23), is employed in Ref. 24 for the study of the thermodynamic limit of the reduced Hartree-Fock functional, in Refs. 20 and 21 in the context of Kohn-Sham density functional theory, as well as in Refs. 16 and 25, where Solovej proves the ionization conjecture within the Hartree-Fock and reduced Hartree-Fock theory of atoms.

To be more specific, to any wave function $\Psi \in \mathcal{W}$ we associate the corresponding $N$-body spin-density matrix $\Gamma_{\Psi}$, defined as the self-adjoint operator on $\bigwedge_{j=1}^{N} L^{2}\left(\mathbb{R}^{3}, \mathbb{C}^{2}\right)$, whose kernel is given by:

$$
\Gamma_{\Psi}\left(\mathbf{x}_{1}, \cdots, \mathbf{x}_{N} ; \mathbf{y}_{1}, \cdots, \mathbf{y}_{N}\right)=\Psi\left(\mathbf{x}_{1}, \cdots, \mathbf{x}_{N}\right) \cdot \bar{\Psi}\left(\mathbf{y}_{1}, \cdots, \mathbf{y}_{N}\right)
$$

We define the set of pure-state $N$-body spin-density matrices as:

$$
\mathcal{M}_{\text {pure }}=\left\{\Gamma_{\Psi}: \Psi \in \mathcal{W}\right\}
$$


Hartree-Fock Theory with a Self-Generated Magnetic Field

Analogously, the set of Slater-state $N$-body spin-density matrices is defined as:

$$
\mathcal{M}_{\text {Slater }}=\left\{\Gamma_{\Psi}: \Psi \in \mathcal{W}_{\text {Slater }}\right\} .
$$

The convex hull of $\mathcal{M}_{\text {pure }}$ is the set of mixed-state $N$-body spin-density matrices, defined as

$$
\mathcal{M}_{\text {mixed }}=\left\{\sum_{k=1}^{+\infty} \lambda_{k} \Gamma_{\Psi_{k}}: 0 \leq \lambda_{k} \leq 1, \sum_{k=1}^{+\infty} \lambda_{k}=1, \Psi_{k} \in \mathcal{W}\right\}
$$

and, as shown by Coleman in Ref. 26, it coincides with the convex hull of $\mathcal{M}_{\text {Slater }}$. For every $\Gamma \in \mathcal{M}_{\text {mixed }}$, we define the associated 1-body spin-density matrix:

$$
\gamma_{\Gamma}=\left(\begin{array}{cc}
\gamma_{\Gamma}^{++} & \gamma_{\Gamma}^{+-} \\
\gamma_{\Gamma}^{-+} & \gamma_{\Gamma}^{--}
\end{array}\right),
$$

where for $a, b=+/-$, we have:

$$
\gamma_{\Gamma}^{a b}(\mathbf{x}, \mathbf{y})=N \sum_{\tau \in\left\{-\frac{1}{2}, \frac{1}{2}\right\}^{N-1}} \int_{\mathbb{R}^{3(N-1)}} \Gamma(\mathbf{x}, a, \mathbf{z}, \tau ; \mathbf{y}, b, \mathbf{z}, \tau) d \mathbf{z} .
$$

We denote the set of mixed-state 1-body spin-density matrices by $\mathcal{D}=\left\{\gamma_{\Gamma}: \Gamma \in \mathcal{M}_{\text {mixed }}\right\}$. In Ref. 26, Coleman proves that:

$$
\mathcal{D}=\left\{\gamma \in \mathcal{S}\left(L^{2}\left(\mathbb{R}^{3}, \mathbb{C}^{2}\right)\right): 0 \leq \gamma \leq 1 ; \operatorname{Tr}(\gamma)=N ; \operatorname{Tr}(-\Delta \gamma)<+\infty\right\}
$$

where $\mathcal{S}\left(L^{2}\left(\mathbb{R}^{3}, \mathbb{C}^{2}\right)\right)$ is the space of self-adjoint operators on $L^{2}\left(\mathbb{R}^{3}, \mathbb{C}^{2}\right)$. In the equation above, we write $\operatorname{Tr}(-\Delta \gamma)$ for $\operatorname{Tr}(|\nabla| \gamma|\nabla|)=\operatorname{Tr}\left((-\Delta)^{1 / 2} \gamma(-\Delta)^{1 / 2}\right)$. The subset of $\mathcal{D}$ given by the 1-body spin-density matrices associated to Slater determinants, instead, is equal to the set of rank- $N$ projections:

$$
\mathcal{P}=\left\{\gamma_{\Gamma}: \Gamma \in \mathcal{M}_{\text {Slater }}\right\}=\left\{\gamma \in \mathcal{D}: \gamma^{2}=\gamma\right\}
$$

Note that, as a consequence of the Spectral theorem (see Chapter VII of Ref. 27), every $\gamma \in \mathcal{D}$ can be diagonalized in an orthonormal basis $\left\{\phi_{k}\right\}_{k \in \mathbb{N}}$ of $L^{2}\left(\mathbb{R}^{3}, \mathbb{C}^{2}\right)$. In other words, we have that:

$$
\gamma=\sum_{k=1}^{+\infty} \lambda_{k} \phi_{k} \otimes \phi_{k}
$$

with $0 \leq \lambda_{k} \leq 1, \sum_{k=1}^{+\infty} \lambda_{k}=N, \phi_{k}=\left(\phi_{k}^{+} ; \phi_{k}^{-}\right)^{T} \in L^{2}\left(\mathbb{R}^{3} ; \mathbb{C}^{2}\right) ;\left(\phi_{k}, \phi_{\ell}\right)_{L^{2}\left(\mathbb{R}^{3}, \mathbb{C}^{2}\right)}=\delta_{k \ell}$ and

$$
\operatorname{Tr}(-\Delta \gamma)=\sum_{k=1}^{+\infty} \lambda_{k}\left\|\nabla \phi_{k}\right\|_{L^{2}}^{2}
$$


Clearly, $\phi_{k}$ are eigenfunctions of $\gamma$ with corresponding eigenvalues $\lambda_{k}$ and the components of $\gamma$ have the form:

$$
\gamma^{a b}(\mathbf{x}, \mathbf{y})=\sum_{k=1}^{+\infty} \lambda_{k} \phi_{k}^{a}(\mathbf{x}) \overline{\phi_{k}^{b}(\mathbf{y})}
$$

where $a, b=+/-$.

Simple algebraic calculations imply that for any Slater determinant $\Psi=\frac{1}{\sqrt{N !}} \operatorname{det}\left(\psi_{\mathrm{k}}\right)$, the following identity follows:

$$
\mathcal{E}_{\mathrm{SMHF}}\left(\psi_{1}, \cdots, \psi_{N}, \mathbf{A}\right)=\mathcal{E}_{\mathrm{SM}}(\Psi, \mathbf{A})=\operatorname{Tr}\left(H_{\mathrm{SM}} \Gamma_{\Psi}\right)=\mathcal{E}_{\mathrm{SMHF}}\left(\gamma_{\Gamma_{\Psi}}, \mathbf{A}\right),
$$

where we define:

$$
\begin{aligned}
\mathcal{E}_{\mathrm{SMHF}}\left(\gamma_{\Gamma_{\Psi}}, \mathbf{A}\right) & =\frac{1}{2} \operatorname{Tr}\left(\sigma \cdot(i \nabla-\mathbf{A}){\gamma_{\Gamma_{\Psi}}}(i \nabla-\mathbf{A}) \cdot \sigma\right)+\int_{\mathbb{R}^{3}} v \rho_{\gamma_{\Gamma_{\Psi}}} \\
& +\frac{1}{2} \iint_{\mathbb{R}^{3} \times \mathbb{R}^{3}} \frac{\rho_{\gamma_{\Gamma_{\Psi}}}(\mathbf{x}) \rho_{\gamma_{\Gamma_{\Psi}}}(\mathbf{y})-\operatorname{Tr}_{\mathbb{C}^{2}}\left(\left|\gamma_{\Gamma_{\Psi}}(\mathbf{x}, \mathbf{y})\right|^{2}\right)}{|\mathbf{x}-\mathbf{y}|} d \mathbf{x} d \mathbf{y} \\
& +\frac{1}{\alpha^{2}} \int_{\mathbb{R}^{3}}|\nabla \times \mathbf{A}|^{2}
\end{aligned}
$$

and

$$
\rho_{{\gamma_{\Gamma_{\Psi}}}}=\rho_{{\gamma_{\Gamma_{\Psi}}}}^{+}(\mathbf{x})+\rho_{{\gamma_{\Gamma_{\Psi}}}^{-}}^{-}(\mathbf{x})=\sum_{k=1}^{+\infty} \lambda_{k}\left|\phi_{k}^{+}(\mathbf{x})\right|^{2}+\sum_{k=1}^{+\infty} \lambda_{k}\left|\phi_{k}^{-}(\mathbf{x})\right|^{2}
$$

is the total electronic density associated to $\gamma_{\Gamma_{\Psi}}$, with $\gamma_{\Gamma_{\Psi}}$ decomposed according to (10). Note that the definition of the energy (11) can be extended to mixed-states. In other words, (11) is well defined for any $(\gamma, \mathbf{A})$ such that:

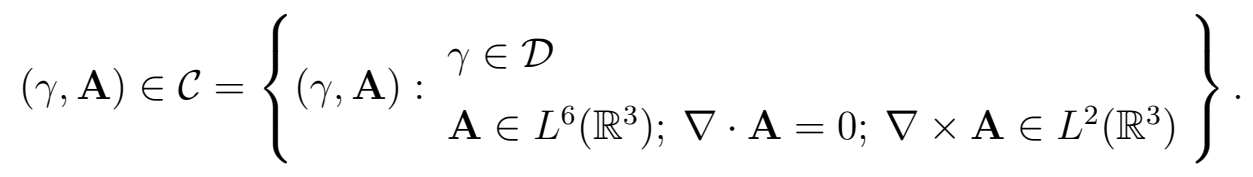

Moreover, Lieb's variational principle (see Ref. 28) implies that minimizing over mixedstates or rank- $N$ projections does not change the value of the ground state. A simple proof of this is given by Bach in Ref. 29 and it can be easily adapted to the spin-magnetic case. Hence, it is clear that recasting problem (6) in terms of the 1-body spin-density matrices leads us to the following variational problem:

$$
E_{\mathrm{SMHF}}=\inf \left\{\mathcal{E}_{\mathrm{SMHF}}(\gamma, \mathbf{A}):(\gamma, \mathbf{A}) \in \mathcal{C}\right\}
$$

The main result of this paper is the following theorem, whose proof is outlined in the next section: 
Hartree-Fock Theory with a Self-Generated Magnetic Field

Theorem I.1 (Existence of HF Minimizers : Spin-Polarized Case). If each nuclear charge satisfies $Z_{k} \alpha^{2} \leq 0.052$ and $N \leq Z=\sum_{k=1}^{K} Z_{k}$, then there exists a minimizer $(\gamma, \mathbf{A}) \in$ $\mathcal{C}$ for $(15)$.

Remark I.2. The condition $Z_{k} \alpha^{2} \leq 0.052$ is precisely the condition for magnetic stability derived in Ref. 4. This means $Z_{k} \leq 975$, which includes all elements in the periodic table.

Using similar ideas to those employed in the proof of Theorem I.1, we can also prove existence of Hartree-Fock minimizers for spinless Coulomb systems interacting with selfgenerated magnetic fields, as provided by the following theorem:

Theorem I.3 (Existence of HF Minimizers: Spinless Case). If $N \leq Z=\sum_{k=1}^{K} Z_{k}$, then there exists a minimizer $(\gamma, \mathbf{A}) \in \mathcal{C}$ for the spinless counterpart of Problem (15):

$$
E_{\mathrm{MHF}}=\inf \left\{\mathcal{E}_{\mathrm{MHF}}(\gamma, \mathbf{A}):(\gamma, \mathbf{A}) \in \mathcal{C}\right\}
$$

where

$$
\begin{aligned}
\mathcal{E}_{\mathrm{MHF}}(\gamma, \mathbf{A}) & =\frac{1}{2} \operatorname{Tr}((i \nabla-\mathbf{A}) \gamma(i \nabla-\mathbf{A}))+\int_{\mathbb{R}^{3}} v \rho_{\gamma} \\
& +\frac{1}{2} \iint_{\mathbb{R}^{3} \times \mathbb{R}^{3}} \frac{\rho_{\gamma}(\mathbf{x}) \rho_{\gamma}(\mathbf{y})-\operatorname{Tr}_{\mathbb{C}^{2}}\left(|\gamma(\mathbf{x}, \mathbf{y})|^{2}\right)}{|\mathbf{x}-\mathbf{y}|} d \mathbf{x} d \mathbf{y} \\
& +\frac{1}{\alpha^{2}} \int_{\mathbb{R}^{3}}|\nabla \times \mathbf{A}|^{2} .
\end{aligned}
$$

Remark I.4. Note that, in the spinless case, we do not need to impose the condition for magnetic stability on the single nuclear charges and the theorem holds true for any neutral or positively charged system.

The article is organized as follows: in Section II, we prove Theorem I.1. Our proof is based on variational techniques applied to the Hartree-Fock energy functional. In particular, it relies on the fact that we can relax the trace constraint on the density matrix $\gamma$, solve the relaxed problem using the direct method of calculus of variation and finally show that the minimizer to the relaxed problem has in fact the right trace (i.e. $\operatorname{Tr}(\gamma)=N$ ). This last fact is a consequence of Lemma II.15 that characterizes the eigenvalues of the operator defined by the Euler-Lagrange equations associated to (15). This Lemma will be proved in Section III. Theorem I.3 follows directly from the proof of Theorem I.1. 
Hartree-Fock Theory with a Self-Generated Magnetic Field

\section{PROOF OF THEOREM I.1}

As we have seen, the first step in our proof is to relax the trace constraint. In the following lemma we prove that the ground state is not affected by this relaxation.

Lemma II.1. The following equality holds

$$
E_{\mathrm{SMHF}}=E_{\mathrm{SMHF}}^{\mathrm{R}},
$$

where

$$
E_{\mathrm{SMHF}}^{\mathrm{R}}=\inf \left\{\mathcal{E}_{\mathrm{SMHF}}(\gamma, \mathbf{A}):(\gamma, \mathbf{A}) \in \mathcal{C}^{\mathrm{R}}\right\}
$$

is the relaxed problem,

$$
\mathcal{C}^{\mathrm{R}}= \begin{cases}\left.(\gamma, \mathbf{A}): \begin{array}{l}
\gamma \in \mathcal{D}^{\mathrm{R}} \\
\mathbf{A} \in L^{6}\left(\mathbb{R}^{3}\right) ; \nabla \cdot \mathbf{A}=0 ; \nabla \times \mathbf{A} \in L^{2}\left(\mathbb{R}^{3}\right)
\end{array}\right\}\end{cases}
$$

and

$$
\mathcal{D}^{\mathrm{R}}=\left\{\gamma \in \mathcal{S}\left(L^{2}\left(\mathbb{R}^{3}, \mathbb{C}^{2}\right)\right): 0 \leq \gamma \leq 1 ; \operatorname{Tr}(\gamma) \leq N ; \operatorname{Tr}(\nabla \gamma \nabla)<+\infty\right\}
$$

Remark II.2. Given two Hermitian matrices $\mathbf{R}, \mathbf{S} \in \mathbf{C}^{N \times N}$, we say that $\mathbf{R} \leq \mathbf{S}$ if $(\mathbf{R} \xi, \xi)_{\mathbb{C}^{N}} \leq(\mathbf{S} \xi, \xi)_{\mathbb{C}^{N}}$ for all $\xi \in \mathbb{C}^{N}$

Proof. We begin by noticing that we can replace $\mathcal{C}^{\mathrm{R}}$ in $(20)$ by:

$$
\mathcal{C}_{0}^{\mathrm{R}}=\left\{(\gamma, \mathbf{A}):(\gamma, \mathbf{A}) \in \mathcal{C}^{\mathrm{R}} ; \operatorname{Ran}(\gamma) \text { is finite; } \operatorname{Ran}(\gamma) \subset \mathrm{C}_{0}^{\infty}\left(\mathbb{R}^{3}, \mathbb{C}^{2}\right)\right\}
$$

where $C_{0}^{\infty}\left(\mathbb{R}^{3}, \mathbb{C}^{2}\right)$ denotes the space of smooth functions with compact support. This follows from the fact that finite rank operators are dense in $\mathcal{D}^{\mathrm{R}} ; C_{0}^{\infty}\left(\mathbb{R}^{3}, \mathbb{C}^{2}\right)$ is dense in $H^{1}\left(\mathbb{R}^{3}, \mathbb{C}^{2}\right)$ and functional (4) is continuous in the strong topology of $\mathcal{D}^{\mathrm{R}}$.

It is clear that $E_{\mathrm{SMHF}}^{\mathrm{R}} \leq E_{\mathrm{SMHF}}$ since we are minimizing on a larger class of admissible functions. Thus, we just need to prove that for every $(\gamma, \mathbf{A}) \in \mathcal{C}_{0}^{\mathrm{R}}$,

$$
E_{\mathrm{SMHF}} \leq \mathcal{E}_{\mathrm{SMHF}}(\gamma, \mathbf{A})
$$

Fix $(\gamma, \mathbf{A}) \in \mathcal{C}_{0}^{\mathrm{R}}$ arbitrarily. Define $\mu=N-\operatorname{Tr}(\gamma) \geq 0$. Since $\gamma$ is finite rank and compact support, it can be decomposed as:

$$
\gamma=\sum_{k=1}^{N_{\gamma}} n_{k} \psi_{k} \otimes \psi_{k}
$$


Hartree-Fock Theory with a Self-Generated Magnetic Field

where $\psi_{k}(\mathbf{x})=\left(\psi_{k}^{+}(\mathbf{x}) ; \psi_{k}^{-}(\mathbf{x})\right)^{T} ; \psi_{k}^{+}, \psi_{k}^{-}$compact support; $\left(\psi_{k} ; \psi_{\ell}\right)_{L^{2}\left(\mathbb{R}^{3}, \mathbb{C}^{2}\right)}=\delta_{k \ell}$.

Since $\left\{\psi_{k}^{+/-}\right\}_{k=1}^{N_{\gamma}} \in C_{0}^{\infty}\left(\mathbb{R}^{3}\right)$, we can find a compact set $\Omega \subset \mathbb{R}^{3}$ such that $\operatorname{supp}\left(\psi_{k}^{+/-}\right) \subset \Omega$ for all $k=1, \ldots, N_{\gamma}$. For any given $\delta>0$, consider $\phi_{k}(\mathbf{x})=\left(\phi_{k}^{+}(\mathbf{x}) ; \phi_{k}^{-}(\mathbf{x})\right)^{T}$ where $\phi_{k}^{+/-}$are smooth functions with compact support such that $\operatorname{supp}\left(\phi_{k}^{+/-}\right) \subset \Omega^{c}$ for all $k=1, \ldots, N_{\gamma}$; $\left(\phi_{k}, \phi_{\ell}\right)_{L^{2}\left(\mathbb{R}^{3}, \mathbb{C}^{2}\right)}=\delta_{k \ell}$ and such that if we define:

$$
\widetilde{\gamma}=\sum_{k=1}^{N_{\gamma}} \widetilde{n}_{k} \phi_{k} \otimes \phi_{k}
$$

with the coefficients $\widetilde{n}_{k}$ conveniently chosen, then $\operatorname{Tr}(\widetilde{\gamma})=\mu$.

Moreover, we require that:

$$
\mathcal{L}=\frac{1}{2} \operatorname{Tr}(\sigma \cdot(i \nabla-\mathbf{A}) \widetilde{\gamma}(i \nabla-\mathbf{A}) \cdot \sigma)+\frac{1}{2} \iint_{\mathbb{R}^{3} \times \mathbb{R}^{3}} \frac{\rho_{\widetilde{\gamma}}(\mathbf{x}) \rho_{\widetilde{\gamma}}(\mathbf{y})-\operatorname{Tr}_{\mathbb{C}^{2}}|\widetilde{\gamma}(\mathbf{x}, \mathbf{y})|^{2}}{|\mathbf{x}-\mathbf{y}|} d \mathbf{x} d \mathbf{y} \leq \delta
$$

Note that if $\mathbf{A}=0$ this can always be achieved by rescaling $\phi_{k}$, and for general $\mathbf{A} \in L^{6}\left(\mathbb{R}^{3}\right)$ this can always be achieved by rescaling and translating $\phi_{k}$.

Let $\mathbf{e}_{0} \in \mathbb{R}^{3}$ be a unit vector such that $\operatorname{supp}\left(\phi_{k}^{+/-}\left(\cdot+n \mathbf{e}_{0}\right)\right) \subset \Omega^{c}$ for all $k=1, \ldots, N_{\gamma}$ and for all $n \in \mathbb{N}$, and define $\psi_{k}^{n}=\frac{1}{\sqrt{2}} \psi_{k}+\frac{1}{\sqrt{2}} \phi_{k}\left(\cdot+n \mathbf{e}_{\mathbf{0}}\right)$ for all $k=1, \ldots, N_{\gamma}$. Define:

$$
\gamma_{n}=\sum_{k=1}^{N_{\gamma}}\left(n_{k}+\tilde{n}_{k}\right) \psi_{k}^{n} \otimes \psi_{k}^{n} .
$$

Note that

$$
\begin{aligned}
\left(\psi_{k}^{n}, \psi_{j}^{n}\right)_{L^{2}\left(\mathbb{R}^{3}, \mathbb{C}^{2}\right)} & =\left(\frac{1}{\sqrt{2}} \psi_{k}+\frac{1}{\sqrt{2}} \phi_{k}\left(\cdot+n \mathbf{e}_{0}\right), \frac{1}{\sqrt{2}} \psi_{j}+\frac{1}{\sqrt{2}} \phi_{j}\left(\cdot+n \mathbf{e}_{0}\right)\right)_{L^{2}\left(\mathbb{R}^{3}, \mathbb{C}^{2}\right)} \\
& =\frac{1}{2}\left(\psi_{k}, \psi_{j}\right)_{L^{2}\left(\mathbb{R}^{3}, \mathbb{C}^{2}\right)}+\frac{1}{2}\left(\phi_{k}\left(\cdot+n \mathbf{e}_{0}\right), \phi_{j}\left(\cdot+n \mathbf{e}_{0}\right)\right)_{L^{2}\left(\mathbb{R}^{3}, \mathbb{C}^{2}\right)} \\
& +\frac{1}{2}\left(\psi_{k} ; \phi_{j}\left(\cdot+n \mathbf{e}_{0}\right)\right)_{L^{2}\left(\mathbb{R}^{3}, \mathbb{C}^{2}\right)}+\frac{1}{2}\left(\phi_{k}\left(\cdot+n \mathbf{e}_{0}\right), \psi_{j}\right)_{L^{2}\left(\mathbb{R}^{3}, \mathbb{C}^{2}\right)} .
\end{aligned}
$$

Since $\phi_{k}$ and $\phi_{j}$ have compact support, the only surviving terms are $\frac{1}{2}\left(\psi_{k}, \psi_{j}\right)_{L^{2}\left(\mathbb{R}^{3}, \mathbb{C}^{2}\right)}$ and $\frac{1}{2}\left(\phi_{k}\left(\cdot+n \mathbf{e}_{0}\right), \phi_{j}\left(\cdot+n \mathbf{e}_{0}\right)\right)_{L^{2}\left(\mathbb{R}^{3}, \mathbb{C}^{2}\right)}=\frac{1}{2}\left(\phi_{k} ; \phi_{j}\right)_{L^{2}\left(\mathbb{R}^{3}, \mathbb{C}^{2}\right)}$. Thus,

$$
\left(\psi_{k}^{n}, \psi_{j}^{n}\right)_{L^{2}\left(\mathbb{R}^{3}, \mathbb{C}^{2}\right)}=\frac{1}{2}\left(\psi_{k}, \psi_{j}\right)_{L^{2}\left(\mathbb{R}^{3}, \mathbb{C}^{2}\right)}+\frac{1}{2}\left(\phi_{k}, \phi_{j}\right)_{L^{2}\left(\mathbb{R}^{3}, \mathbb{C}^{2}\right)}=\delta_{k j}
$$

and $\operatorname{Tr}\left(\gamma_{n}\right)=\sum_{k=1}^{N_{\gamma}}\left(n_{k}+\tilde{n}_{k}\right)=N$. In the same way, since $\mathbf{A} \in L^{6}\left(\mathbb{R}^{3}\right)$ and $\nabla \times \mathbf{A} \in L^{2}\left(\mathbb{R}^{3}\right)$, we have that

$$
\mathcal{E}_{\mathrm{SMHF}}\left(\gamma_{n}, \mathbf{A}\right) \rightarrow \mathcal{L}+\mathcal{E}_{\mathrm{SMHF}}(\gamma, \mathbf{A})
$$


Hartree-Fock Theory with a Self-Generated Magnetic Field

Thus, we obtain that

$$
E_{\mathrm{SMHF}} \leq \mathcal{L}+\mathcal{E}_{\mathrm{SMHF}}(\gamma, \mathbf{A}) \leq \delta+\mathcal{E}_{\mathrm{SMHF}}(\gamma, \mathbf{A})
$$

Since $\delta>0$ is arbitrary, the conclusion follows.

To prove the existence of minimizers for the relaxed problem, we follow the direct method of the calculus of variations. As a first step, we prove that any minimizing sequence of (20) is bounded in an appropriate norm, to be specified later. Hence, we will be able to extract a weakly convergent subsequence, and the existence of minimizers will be established once we prove that functional $\mathcal{E}_{\mathrm{SMHF}}$ is weakly lower semicontinuous. To show boundedness of any minimizing sequence we use the following result, which is an extension of a similar result proved in Ref. 4 in the many-body case:

Lemma II.3. If $Z_{k} \alpha^{2} \leq 0.052$ for $k=1, \ldots, K$, there exist positive constants $K_{1}$ and $K_{2}$, depending solely on $N$ and $\alpha$, such that for every $(\gamma, \mathbf{A}) \in \mathcal{C}$,

$$
\mathcal{E}_{\mathrm{SMHF}}(\gamma, \mathbf{A}) \geq K_{1} \int_{\mathbb{R}^{3}}|\nabla \times \mathbf{A}|^{2}-K_{2}
$$

Proof. From the the proof of Theorem 1, part $(A)$ of Ref. 4, we deduce that there exist positive constants $K_{1}$ and $K_{2}$ such that for any $(\Psi, \mathbf{A}) \in \mathcal{D}_{\mathrm{SM}}$, the following inequality holds:

$$
\mathcal{E}_{\mathrm{SM}}(\Psi, \mathbf{A}) \geq K_{1} \int_{\mathbb{R}^{3}}|\nabla \times \mathbf{A}|^{2}-K_{2}
$$

Moreover, for any $(\gamma, \mathbf{A}) \in \mathcal{C}$, we have that $\gamma \in \mathcal{D}$ and the proof of Lieb's variational principle (see Ref. 28) implies that there exists $\Gamma \in \mathcal{M}_{\text {mixed }}$ such that $\gamma=\gamma_{\Gamma}$ and $\operatorname{Tr}\left(H_{\mathrm{SM}} \Gamma\right) \leq$ $\mathcal{E}_{\mathrm{SMHF}}(\gamma, \mathbf{A})$.

On the other hand, by the definition of $\mathcal{M}_{\text {mixed }}$, we know that $\Gamma=\sum_{k=1}^{+\infty} \lambda_{k} \Psi_{k} \otimes \Psi_{k}$, where $0 \leq \lambda_{k} \leq 1, \sum_{k=1}^{+\infty} \lambda_{k}=1$ and $\Psi_{k} \in \mathcal{W}$.

Hence, the following inequalities hold:

$$
\begin{aligned}
\mathcal{E}_{\mathrm{SMHF}}(\gamma, \mathbf{A}) \geq \operatorname{Tr}\left(H_{\mathrm{SM}} \Gamma\right) & =\sum_{k=1}^{+\infty} \lambda_{k}\left(\Psi_{k}, H_{\mathrm{SM}} \Psi_{k}\right)_{L^{2}\left(\mathbb{R}^{3 N}, \mathbb{C}^{2^{N}}\right)} \\
& =\sum_{k=1}^{+\infty} \lambda_{k} \mathcal{E}_{\mathrm{SM}}\left(\Psi_{k}, \mathbf{A}\right) \geq K_{1} \int_{\mathbb{R}^{3}}|\nabla \times \mathbf{A}|^{2}-K_{2},
\end{aligned}
$$

as claimed. 
Hartree-Fock Theory with a Self-Generated Magnetic Field

We are now able to prove the following lemma, which together with Lemma II.3 establishes the coercivity of functional $\mathcal{E}_{\mathrm{SMHF}}$ in $\mathcal{C}$ :

Lemma II.4. Assume that $Z_{k} \alpha^{2} \leq 0.052$ for $k=1, \ldots, K$. Then, there exists a positive constant $K_{3}>0$ such that if $\left(\gamma_{n}, \mathbf{A}_{n}\right) \in \mathcal{C}$ is a minimizing sequence for (20), then

$$
\mathcal{E}_{\mathrm{SMHF}}\left(\gamma_{n}, \mathbf{A}_{n}\right) \geq \frac{1}{4} \operatorname{Tr}\left(\left(i \nabla-\mathbf{A}_{n}\right) \gamma_{n}\left(i \nabla-\mathbf{A}_{n}\right)\right)-K_{3} .
$$

Proof. Since the ground state energy $E_{\mathrm{SM}}$ is always a lower bound for $E_{\mathrm{SMHF}}$, it follows from the magnetic stability result proved in Ref. 4 that $E_{\mathrm{SMHF}} \geq E_{\mathrm{SM}}>-\infty$.

Consider a minimizing sequence $\left(\gamma_{n}, \mathbf{A}_{n}\right)_{n \in \mathbb{N}} \in \mathcal{C}$ for $(20)$, that is, for every $n \in \mathbb{N},\left(\gamma_{n}, \mathbf{A}_{n}\right) \in$ $\mathcal{C}$ and

$$
\lim _{n \rightarrow+\infty} \mathcal{E}_{\mathrm{SMHF}}\left(\gamma_{n}, \mathbf{A}_{n}\right)=E_{\mathrm{SMHF}} .
$$

Note that, given Lemma II.1, it is always possible to find a minimizing sequence for (20) that is in $\mathcal{C}$ rather than $\mathcal{C}^{\mathrm{R}}$. Since $\left(\gamma_{n}, \mathbf{A}_{n}\right) \in \mathcal{C}$, as a consequence of the Spectral Theorem (see Chapter VII of Ref. 27) we can write:

$$
\gamma_{n}(\mathbf{x}, \mathbf{y})=\left(\begin{array}{cc}
\gamma_{n}^{++}(\mathbf{x}, \mathbf{y}) & \gamma_{n}^{+-}(\mathbf{x}, \mathbf{y}) \\
\gamma_{n}^{-+}(\mathbf{x}, \mathbf{y}) & \gamma_{n}^{--}(\mathbf{x}, \mathbf{y})
\end{array}\right)
$$

where

$$
\gamma_{n}=\sum_{k=1}^{+\infty} \lambda_{k}^{n} \phi_{k}^{n} \otimes \phi_{k}^{n}
$$

and $0 \leq \lambda_{k}^{n} \leq 1, \sum_{k=1}^{+\infty} \lambda_{k}^{n}=N ; \phi_{k}^{n}=\left(\phi_{k}^{n+} ; \phi_{k}^{n-}\right)^{T} \in L^{2}\left(\mathbb{R}^{3} ; \mathbb{C}^{2}\right) ;\left(\phi_{k}^{n} ; \phi_{\ell}^{n}\right)_{L^{2}\left(\mathbb{R}^{3} ; \mathbb{C}^{2}\right)}=\delta_{k \ell}$.

Each component of $\gamma_{n}$ is given by:

$$
\gamma_{n}^{a b}(\mathbf{x}, \mathbf{y})=\sum_{k=1}^{+\infty} \lambda_{k}^{n} \phi_{k}^{n a}(\mathbf{x}) \overline{\phi_{k}^{n b}(\mathbf{y})}
$$

where $a, b=+/-$.

Given that $\left(\gamma_{n}, \mathbf{A}_{n}\right)$ is a minimizing sequence and the ground state energy is finite, it follows that $\mathcal{E}_{\mathrm{SMHF}}\left(\gamma_{n}, \mathbf{A}_{n}\right)$ is a bounded sequence, which means that $\exists M_{1}>0$ such that $\left|\mathcal{E}_{\mathrm{SMHF}}\left(\gamma_{n}, \mathbf{A}_{n}\right)\right| \leq M_{1}$ for every $n$. As a consequence of Lemma II.3, we get that for every $n$

$$
M_{1} \geq \mathcal{E}_{\mathrm{SMHF}}\left(\gamma_{n}, \mathbf{A}_{n}\right) \geq K_{1} \int_{\mathbb{R}^{3}}\left|\nabla \times \mathbf{A}_{n}\right|^{2}-K_{2}
$$

This implies that $\left\{\nabla \times \mathbf{A}_{n}\right\}_{n \in \mathbb{N}}$ is uniformly bounded in $L^{2}\left(\mathbb{R}^{3}\right): \exists M_{2}>0$ such that

$$
\left\|\nabla \times \mathbf{A}_{n}\right\|_{L^{2}} \leq M_{2} \quad \forall n \in \mathbb{N}
$$


Hartree-Fock Theory with a Self-Generated Magnetic Field

Besides, simple algebraic calculations provide that, for any $(\gamma, \mathbf{A}) \in \mathcal{C}$, it holds:

$$
\operatorname{Tr}(\sigma \cdot(i \nabla-\mathbf{A}) \gamma(i \nabla-\mathbf{A}) \cdot \sigma)=\operatorname{Tr}((i \nabla-\mathbf{A}) \gamma(i \nabla-\mathbf{A}))-\int_{\mathbb{R}^{3}} \mathbf{m}_{\gamma} \cdot(\nabla \times \mathbf{A}),
$$

where $\mathbf{m}_{\gamma}=\operatorname{Tr}_{\mathbb{C}^{2}}\left(\sigma \cdot R_{\gamma}\right)$ is the spin angular momentum density, $R_{\gamma}(\mathbf{x}) \in \mathcal{M}_{2 \times 2}(\mathbb{C})$ is the electronic spin-density matrix associated to $\gamma$ and the last term in (26) is the direct coupling. For $\gamma$ trace class, the $2 \times 2$ matrix

$$
R_{\gamma}(\mathbf{x})=\left(\begin{array}{cc}
\rho_{\gamma}^{++}(\mathbf{x}) & \rho_{\gamma}^{+-}(\mathbf{x}) \\
\rho_{\gamma}^{-+}(\mathbf{x}) & \rho_{\gamma}^{--}(\mathbf{x})
\end{array}\right)
$$

is defined through the formula

$$
\rho_{\gamma}^{a b}(\mathbf{x})=\sum_{k=1}^{+\infty} \lambda_{k} \phi_{k}^{a}(\mathbf{x}) \overline{\phi_{k}^{b}(\mathbf{x})}
$$

with $a, b=+/-$ and where $\gamma$ is decomposed according to (10). The total electronic density associated to $\gamma$ is defined as:

$$
\rho_{\gamma}(\mathbf{x})=\rho_{\gamma}^{++}(\mathbf{x})+\rho_{\gamma}^{--}(\mathbf{x})=\sum_{k=1}^{+\infty} \lambda_{k}\left|\phi_{k}^{+}(\mathbf{x})\right|^{2}+\sum_{k=1}^{+\infty} \lambda_{k}\left|\phi_{k}^{-}(\mathbf{x})\right|^{2} .
$$

As a consequence of (26), we can write:

$$
\begin{aligned}
\mathcal{E}_{\mathrm{SMHF}}\left(\gamma_{n}, \mathbf{A}_{n}\right) & =\frac{1}{2} \operatorname{Tr}\left(\sigma \cdot\left(i \nabla-\mathbf{A}_{n}\right) \gamma_{n}\left(i \nabla-\mathbf{A}_{n}\right) \cdot \sigma\right)+\int_{\mathbb{R}^{3}} v \rho_{\gamma_{n}} \\
& +\frac{1}{2} \iint_{\mathbb{R}^{3} \times \mathbb{R}^{3}} \frac{\rho_{\gamma_{n}}(\mathbf{x}) \rho_{\gamma_{n}}(\mathbf{y})-\operatorname{Tr}_{\mathbb{C}^{2}}\left(\left|\gamma_{n}(\mathbf{x}, \mathbf{y})\right|^{2}\right)}{|\mathbf{x}-\mathbf{y}|} d \mathbf{x} d \mathbf{y} \\
& +\frac{1}{\alpha^{2}} \int_{\mathbb{R}^{3}}\left|\nabla \times \mathbf{A}_{n}\right|^{2} \\
& =\frac{1}{2} \operatorname{Tr}\left(\left(i \nabla-\mathbf{A}_{n}\right) \gamma_{n}\left(i \nabla-\mathbf{A}_{n}\right)\right)-\frac{1}{2} \int_{\mathbb{R}^{3}} \mathbf{m}_{\gamma_{n}} \cdot\left(\nabla \times \mathbf{A}_{n}\right)+\int_{\mathbb{R}^{3}} v \rho_{\gamma_{n}} \\
& +\frac{1}{2} \iint_{\mathbb{R}^{3} \times \mathbb{R}^{3}} \frac{\rho_{\gamma_{n}}(\mathbf{x}) \rho_{\gamma_{n}}(\mathbf{y})-\operatorname{Tr}_{\mathbb{C}^{2}}\left(\left|\gamma_{n}(\mathbf{x}, \mathbf{y})\right|^{2}\right)}{|\mathbf{x}-\mathbf{y}|} d \mathbf{x} d \mathbf{y} \\
& +\frac{1}{\alpha^{2}} \int_{\mathbb{R}^{3}}\left|\nabla \times \mathbf{A}_{n}\right|^{2} .
\end{aligned}
$$

We consider each term in the energy separately. First, note that Cauchy-Schwarz inequality for series implies that

$$
\operatorname{Tr}_{\mathbb{C}^{2}}\left(|\gamma(\mathbf{x}, \mathbf{y})|^{2}\right) \leq \rho_{\gamma}(\mathbf{x}) \rho_{\gamma}(\mathbf{y})
$$

and therefore:

$$
\frac{1}{2} \iint_{\mathbb{R}^{3} \times \mathbb{R}^{3}} \frac{\rho_{\gamma_{n}(\mathbf{x})} \rho_{\gamma_{n}}(\mathbf{y})-\operatorname{Tr}_{\mathbb{C}^{2}}\left|\gamma_{n}(\mathbf{x}, \mathbf{y})\right|^{2}}{|\mathbf{x}-\mathbf{y}|} \geq 0
$$


Hartree-Fock Theory with a Self-Generated Magnetic Field

As it concerns the direct coupling term, the Cauchy-Schwarz inequality provides that

$$
\left|\frac{1}{2} \int_{\mathbb{R}^{3}} \mathbf{m}_{\gamma_{n}} \cdot \nabla \times \mathbf{A}_{n}\right| \leq\left\|\mathbf{m}_{\gamma_{n}}\right\|_{L^{2}}\left\|\nabla \times \mathbf{A}_{n}\right\|_{L^{2}} \leq\left\|\mathbf{m}_{\gamma_{n}}\right\|_{L^{2}} M_{2},
$$

where the last inequality follows from (25).

By definition, we have that

$$
\mathbf{m}_{\gamma_{n}}=\operatorname{Tr}_{\mathbb{C}^{2}}\left(\sigma \cdot R_{\gamma_{n}}\right)=\left(\begin{array}{c}
\rho_{\gamma_{n}}^{-+}+\rho_{\gamma_{n}}^{+-} \\
i \rho_{\gamma_{n}}^{+-}-i \rho_{\gamma_{n}}^{-+} \\
\rho_{\gamma_{n}}^{++}-\rho_{\gamma_{n}}^{--}
\end{array}\right)
$$

and, consequently, that

$$
\left\|\mathbf{m}_{\gamma_{n}}\right\|_{L^{2}}^{2} \leq 4\left\|\rho_{\gamma_{n}}^{-+}\right\|_{L^{2}}^{2}+2\left\|\rho_{\gamma_{n}}^{++}\right\|_{L^{2}}^{2}+2\left\|\rho_{\gamma_{n}}^{--}\right\|_{L^{2}}^{2}
$$

where (31) follows from the fact that $\rho_{\gamma_{n}}^{-+}=\overline{\rho_{\gamma_{n}}^{+-}}$and from the standard inequality

$$
-2 \rho_{\gamma_{n}}^{++} \rho_{\gamma_{n}}^{--} \leq\left(\rho_{\gamma_{n}}^{++}\right)^{2}+\left(\rho_{\gamma_{n}}^{--}\right)^{2}
$$

Given (31), it is clear that, to control the direct coupling term, we need to control the $L^{2}$ norm of $\rho_{\gamma_{n}}^{a b}$. In particular, we have the following bound, which is an extension of Lemma 1 in Ref. 20:

Lemma II.5. For all $1 \leq p \leq 3, \exists C_{p}>0$ constant such that for all $(\gamma, \mathbf{A}) \in \mathcal{C}$ and $a, b=+/-$, it holds

$$
\left\|\rho_{\gamma}^{a b}\right\|_{L^{p}} \leq C_{p} N^{\frac{3-p}{2 p}} \operatorname{Tr}((i \nabla-\mathbf{A}) \gamma(i \nabla-\mathbf{A}))^{\frac{3(p-1)}{2 p}}
$$

Proof. First, note that, for $a=+/-$ and $\gamma$ decomposed as $\gamma=\sum_{k=1}^{+\infty} \lambda_{k} \phi_{k} \otimes \phi_{k}$, it holds:

$$
\begin{aligned}
\left|\nabla \rho_{\gamma}^{a a}\right|^{2} & \leq 2\left(\sum_{k=1}^{+\infty} \lambda_{k}\left|\phi_{k}^{a}\right|^{2}\right)\left(\sum_{k=1}^{+\infty} \lambda_{k}|\nabla| \phi_{k}^{a}||^{2}\right) \\
& \leq 4 \rho_{\gamma}^{a a}\left(\sum_{k=1}^{+\infty} \lambda_{k}\left|(i \nabla-\mathbf{A}) \phi_{k}^{a}\right|^{2}\right),
\end{aligned}
$$

where (33) follows from the discrete Cauchy-Schwarz inequality and (34) follows from the diamagnetic inequality. Integrating on both sides leads to

$$
\left\|\nabla \sqrt{\rho_{\gamma}^{a a}}\right\|_{L^{2}}^{2} \leq \operatorname{Tr}\left((i \nabla-\mathbf{A}) \gamma^{a a}(i \nabla-\mathbf{A})\right)
$$


Hartree-Fock Theory with a Self-Generated Magnetic Field

which together with the Sobolev inequality implies that

$$
\left\|\rho_{\gamma}^{a a}\right\|_{L^{3}} \leq M_{3} \operatorname{Tr}\left((i \nabla-\mathbf{A}) \gamma^{a a}(i \nabla-\mathbf{A})\right)
$$

with $M_{3}$ positive constant. In the same way, when $a, b=+/-$, we denote

$$
\rho_{|\gamma|}^{a b}(\mathbf{x})=\sum_{k=1}^{+\infty} \lambda_{k}\left|\phi_{k}^{a}(\mathbf{x})\right|\left|\phi_{k}^{b}(\mathbf{x})\right| .
$$

For this function, analogously to (33), we have

$$
\left|\nabla \rho_{|\gamma|}^{a b}\right| \leq\left(\sum_{k=1}^{+\infty} \lambda_{k}\left(\left|(i \nabla-\mathbf{A}) \phi_{k}^{a}\right|^{2}+\left|(i \nabla-\mathbf{A}) \phi_{k}^{b}\right|^{2}\right)\right)^{1 / 2}\left(\sum_{k=1}^{+\infty} \lambda_{k}\left(\left|\phi_{k}^{a}\right|^{2}+\left|\phi_{k}^{b}\right|^{2}\right)\right)^{1 / 2} .
$$

If we integrate on both sides, apply Hölder's inequality and (35), we obtain

$$
\begin{aligned}
\left\|\nabla \rho_{|\gamma|}^{a b}\right\|_{L^{3 / 2}} & \leq \operatorname{Tr}((i \nabla-\mathbf{A}) \gamma(i \nabla-\mathbf{A}))^{1 / 2}\left\|\rho_{\gamma}^{a a}+\rho_{\gamma}^{b b}\right\|_{L^{3}}^{1 / 2} \\
& \leq M_{4} \operatorname{Tr}((i \nabla-\mathbf{A}) \gamma(i \nabla-\mathbf{A}))
\end{aligned}
$$

with $M_{4}$ positive constant. Furthermore, the Sobolev embedding $W^{1,3 / 2}\left(\mathbb{R}^{3}\right) \hookrightarrow L^{3}\left(\mathbb{R}^{3}\right)$ implies that

$$
\left\|\rho_{|\gamma|}^{a b}\right\|_{L^{3}} \leq M_{5} \operatorname{Tr}((i \nabla-\mathbf{A}) \gamma(i \nabla-\mathbf{A})),
$$

with $M_{5}$ positive constant. Note that, if $a=b, \rho_{|\gamma|}^{a a}=\rho_{\gamma}^{a a}$ and we recover (35). Clearly, $\rho_{|\gamma|}^{a b}$ is also in $L^{1}\left(\mathbb{R}^{3}\right)$, given the inequality

$$
\rho_{|\gamma|}^{a b} \leq \sum_{k=1}^{+\infty} \frac{\lambda_{k}}{2}\left(\left|\phi_{k}^{a}\right|^{2}+\left|\phi_{k}^{b}\right|^{2}\right) \leq \rho_{\gamma}^{a a}+\rho_{\gamma}^{b b}=\rho_{\gamma}
$$

which implies

$$
\left\|\rho_{|\gamma|}^{a b}\right\|_{L^{1}} \leq \operatorname{Tr}(\gamma)=N
$$

As a consequence, we have that (36), (37) and the interpolation inequality provide the following bound, for $1 \leq p \leq 3$,

$$
\left\|\rho_{|\gamma|}^{a b}\right\|_{L^{p}} \leq C_{p} N^{\frac{3-p}{2 p}} \operatorname{Tr}((i \nabla-\mathbf{A}) \gamma(i \nabla-\mathbf{A}))^{\frac{3(p-1)}{2 p}} .
$$

Finally, since $\left|\rho_{\gamma}^{a b}\right| \leq \rho_{|\gamma|}^{a b}$, the desired inequality for $\left\|\rho_{\gamma}^{a b}\right\|_{L^{p}}$ follows.

It is now clear that (29), (31), Lemma II.5 and Young's inequality imply that for every $\eta>0, \exists M_{6}>0$ constant such that

$$
\left|\frac{1}{2} \int_{\mathbb{R}^{3}} \mathbf{m}_{\gamma_{n}} \cdot \nabla \times \mathbf{A}_{n}\right| \leq \eta \operatorname{Tr}\left(\left(i \nabla-\mathbf{A}_{n}\right) \gamma_{n}\left(i \nabla-\mathbf{A}_{n}\right)\right)+M_{6} .
$$


Hartree-Fock Theory with a Self-Generated Magnetic Field

At this point, the only term we still need to control is the potential term. Note that the potential can be decomposed as:

$$
v(\mathbf{x})=-\sum_{k=1}^{K} \frac{Z_{k}}{\left|\mathbf{x}-\mathbf{R}_{k}\right|} \mathbb{1}_{\left\{\left|\mathbf{x}-\mathbf{R}_{k}\right| \leq 1\right\}}(\mathbf{x})-\sum_{k=1}^{K} \frac{Z_{k}}{\left|\mathbf{x}-\mathbf{R}_{k}\right|} \mathbb{1}_{\left\{\left|\mathbf{x}-\mathbf{R}_{k}\right|>1\right\}}(\mathbf{x}),
$$

where, given a set $T, \mathbb{1}_{T}$ denotes its indicator function.

Thus $v \in L^{\frac{3}{2}+\epsilon^{\prime}}\left(\mathbb{R}^{3}\right)+L^{\infty}\left(\mathbb{R}^{3}\right)$, for every $\epsilon^{\prime}>0$. Hölder's inequality implies that

$$
\left|\int_{\mathbb{R}^{3}} v(\mathbf{x}) \rho_{\gamma_{n}}(\mathbf{x}) d \mathbf{x}\right| \leq\|v\|_{L^{\frac{3}{2}+\epsilon^{\prime}}+L^{\infty}}\left\|\rho_{\gamma_{n}}\right\|_{L^{1} \cap L^{3-\epsilon^{\prime}}} .
$$

If we apply (32) with $p=1$ and $p=3-\epsilon^{\prime}$ respectively, we obtain:

$$
\left|\int_{\mathbb{R}^{3}} v(\mathbf{x}) \rho_{\gamma_{n}}(\mathbf{x}) d \mathbf{x}\right| \leq M_{7}\|v\|_{L^{\frac{3}{2}+\epsilon^{\prime}}+L^{\infty}}\left(1+\left(\operatorname{Tr}\left(\left(i \nabla-\mathbf{A}_{n}\right) \gamma_{n}\left(i \nabla-\mathbf{A}_{n}\right)\right)\right)^{\alpha_{1}}\right),
$$

where $M_{7}$ is a positive constant and $0 \leq \alpha_{1}<1$. Again, Young's inequality provides that for every $\eta>0, \exists M_{8}>0$ constant such that:

$$
\left|\int_{\mathbb{R}^{3}} v(\mathbf{x}) \rho_{\gamma_{n}}(\mathbf{x}) d \mathbf{x}\right| \leq \eta \operatorname{Tr}\left(\left(i \nabla-\mathbf{A}_{n}\right) \gamma_{n}\left(i \nabla-\mathbf{A}_{n}\right)\right)+M_{8} .
$$

If we collect the inequalities in (28), (39) and (42), we obtain:

$$
\mathcal{E}_{\mathrm{SMHF}}\left(\gamma_{n}, \mathbf{A}_{n}\right) \geq\left(\frac{1}{2}-2 \eta\right) \operatorname{Tr}\left(\left(i \nabla-\mathbf{A}_{n}\right) \gamma_{n}\left(i \nabla-\mathbf{A}_{n}\right)\right)-K_{3},
$$

where $K_{3}=M_{6}+M_{8}$, and the desired result follows by choosing $\eta<1 / 8$.

As a consequence of Lemmas II.3 and II.4, we get that, given any minimizing sequence $\left\{\left(\gamma_{n}, \mathbf{A}_{n}\right)\right\}_{n \in \mathbb{N}} \in \mathcal{C}$, the sequences $\left\{\nabla \times \mathbf{A}_{n}\right\}_{n \in \mathbb{N}}$ and $\left\{\operatorname{Tr}\left(\left(i \nabla-\mathbf{A}_{n}\right) \gamma_{n}\left(i \nabla-\mathbf{A}_{n}\right)\right)\right\}_{n \in \mathbb{N}}$ are uniformly bounded in $L^{2}\left(\mathbb{R}^{3}\right)$ and $\mathbb{R}$, respectively. By the Gagliardo-Nirenberg inequality and the fact that $\nabla \cdot \mathbf{A}_{n}=0$, we also obtain that:

$$
\left\|\mathbf{A}_{n}\right\|_{L^{6}} \leq C_{G N}\left\|\nabla \times \mathbf{A}_{n}\right\|_{L^{2}} \leq C_{G N} M_{2} \quad \forall n \in \mathbb{N}
$$

which means that $\left\{\mathbf{A}_{n}\right\}_{n \in \mathbb{N}}$ is uniformly bounded in $L^{6}\left(\mathbb{R}^{3}\right)$.

Note that for each $\phi_{k}^{n}$ in (23), it holds that $i \nabla \phi_{k}^{n}=\left(i \nabla-\mathbf{A}_{n}\right) \phi_{k}^{n}+\mathbf{A}_{n} \phi_{k}^{n}$, which implies that

$$
\left\|\nabla \phi_{k}^{n}\right\|_{L^{2}} \leq\left\|\left(i \nabla-\mathbf{A}_{n}\right) \phi_{k}^{n}\right\|_{L^{2}}+\left\|\mathbf{A}_{n} \phi_{k}^{n}\right\|_{L^{2}}
$$


Hartree-Fock Theory with a Self-Generated Magnetic Field

In addition, for every $\eta>0$, there exists a constant $G_{1}>0$, independent of $n$, such that:

$$
\begin{aligned}
\left\|\mathbf{A}_{n} \phi_{k}^{n}\right\|_{L^{2}} & \leq\left\|\mathbf{A}_{n}\right\|_{L^{6}}\left\|\phi_{k}^{n}\right\|_{L^{3}} \\
& \leq\left\|\mathbf{A}_{n}\right\|_{L^{6}}\left\|\phi_{k}^{n}\right\|_{L^{2}}^{1 / 2}\left\|\phi_{k}^{n}\right\|_{L^{6}}^{1 / 2} \\
& \leq C_{\mathrm{GN}}^{1 / 2}\left\|\mathbf{A}_{n}\right\|_{L^{6}}\left\|\nabla \phi_{k}^{n}\right\|_{L^{2}}^{1 / 2} \\
& \leq \eta\left\|\nabla \phi_{k}^{n}\right\|_{L^{2}}+G_{1},
\end{aligned}
$$

where the inequalities above follow, respectively, from Hölder's inequality, interpolation inequality, the fact that $\phi_{k}^{n}$ have unit $L^{2}$-norm and the Sobolev inequality, uniform boundedness of $\left\{\mathbf{A}_{n}\right\}_{n \in \mathbb{N}}$ in $L^{6}\left(\mathbb{R}^{3}\right)$ and Young's inequality. From (45) and (46), we deduce that, for $\eta<1 / 2$,

$$
\frac{1}{2}\left\|\nabla \phi_{k}^{n}\right\|_{L^{2}} \leq\left\|\left(i \nabla-\mathbf{A}_{n}\right) \phi_{k}^{n}\right\|_{L^{2}}+G_{1},
$$

which implies that

$$
\operatorname{Tr}\left(-\Delta \gamma_{n}\right) \leq G_{2} \operatorname{Tr}\left(\left(i \nabla-\mathbf{A}_{n}\right) \gamma_{n}\left(i \nabla-\mathbf{A}_{n}\right)\right)+G_{3},
$$

where $G_{2}, G_{3}$ are positive constants independent of $n$.

Finally, recalling that $\left\{\operatorname{Tr}\left(\left(i \nabla-\mathbf{A}_{n}\right) \gamma_{n}\left(i \nabla-\mathbf{A}_{n}\right)\right)\right\}_{n \in \mathbb{N}}$ is uniformly bounded, we conclude that the sequence $\left\{\gamma_{n}\right\}_{n \in \mathbb{N}}$ is also uniformly bounded in the Banach space:

$$
\mathcal{B}=\left\{\gamma \in \mathcal{S}\left(L^{2}\left(\mathbb{R}^{3}, \mathbb{C}^{2}\right)\right),\|\gamma\|_{\mathcal{B}}=\operatorname{Tr}(|\gamma|)+\operatorname{Tr}(|\nabla| \gamma|\nabla|)<+\infty\right\} .
$$

As a consequence, there are $\boldsymbol{\alpha} \in L^{6}\left(\mathbb{R}^{3}\right)$ such that $\nabla \times \boldsymbol{\alpha} \in L^{2}\left(\mathbb{R}^{3}\right)$ and $\widetilde{\gamma} \in \mathcal{B}$ such that, by passing to a subsequence,

$$
\begin{array}{cr}
\mathbf{A}_{n} \underset{n}{\rightarrow} \boldsymbol{\alpha} & \text { weakly in } L^{6}\left(\mathbb{R}^{3}\right), \\
\nabla \times \mathbf{A}_{n} \underset{n}{\rightarrow} \nabla \times \boldsymbol{\alpha} & \text { weakly in } L^{2}\left(\mathbb{R}^{3}\right), \\
\gamma_{n} \underset{n}{\rightarrow} \widetilde{\gamma} & \text { weakly- } * \text { in } \mathcal{B} .
\end{array}
$$

Furthermore, the Rellich-Kondrachov Theorem, Lemma II.5 and the Banach-Alaoglu Theorem imply that all components of $R_{\gamma_{n}}$ converge to their respective components of $R_{\widetilde{\gamma}}$ strongly in $L_{\text {loc }}^{p}\left(\mathbb{R}^{3}\right)$ for $1 \leq p<3$, weakly in $L^{p}\left(\mathbb{R}^{3}\right)$ for $1 \leq p \leq 3$ and pointwise almost everywhere. A proof of that can be found in Ref. 20. In addition, $\nabla \cdot \boldsymbol{\alpha}=0$ and $\operatorname{Tr}(\widetilde{\gamma}) \leq N$.

In the next lemma we establish the weak lower semicontinuity of functional $\mathcal{E}_{\mathrm{SMHF}}$. This clearly implies that $(\widetilde{\gamma}, \boldsymbol{\alpha}) \in \mathcal{C}^{\mathrm{R}}$ is a minimizer for problem (20). 
Hartree-Fock Theory with a Self-Generated Magnetic Field

Lemma II.6. Functional $\mathcal{E}_{\mathrm{SMHF}}$ is *-weakly lower semicontinous. In other words, given a sequence $\left\{\left(\gamma_{n}, \mathbf{A}_{n}\right)\right\}_{n \in \mathbb{N}} \in \mathcal{C}$ and $(\widetilde{\gamma}, \boldsymbol{\alpha}) \in \mathcal{C}^{\mathrm{R}}$ such that $\gamma_{n} \underset{n}{\stackrel{*}{*}} \widetilde{\gamma}$ weak-* in $\mathcal{B}, \mathbf{A}_{n} \underset{n}{\rightarrow} \boldsymbol{\alpha}$ weakly in $L^{6}\left(\mathbb{R}^{3}\right), \nabla \times \mathbf{A}_{n} \underset{n}{\rightarrow} \nabla \times \boldsymbol{\alpha}$ weakly in $L^{2}\left(\mathbb{R}^{3}\right)$, then

$$
\liminf _{n \rightarrow+\infty} \mathcal{E}_{\mathrm{SMHF}}\left(\gamma_{n}, \mathbf{A}_{n}\right) \geq \mathcal{E}_{\mathrm{SMHF}}(\widetilde{\gamma}, \boldsymbol{\alpha})
$$

Proof. Recall that the energy is given by

$$
\begin{aligned}
\mathcal{E}_{\mathrm{SMHF}}\left(\gamma_{n}, \mathbf{A}\right) & =\frac{1}{2} \operatorname{Tr}\left(\sigma \cdot\left(i \nabla-\mathbf{A}_{n}\right) \gamma_{n}\left(i \nabla-\mathbf{A}_{n}\right) \cdot \sigma\right)+\int_{\mathbb{R}^{3}} v \rho_{\gamma_{n}} \\
& +\frac{1}{2} \iint_{\mathbb{R}^{3} \times \mathbb{R}^{3}} \frac{\rho_{\gamma_{n}}(\mathbf{x}) \rho_{\gamma_{n}}(\mathbf{y})-\operatorname{Tr}_{\mathbb{C}^{2}}\left(\left|\gamma_{n}(\mathbf{x}, \mathbf{y})\right|^{\mathbf{2}}\right)}{|\mathbf{x}-\mathbf{y}|} d \mathbf{x} d \mathbf{y} \\
& +\frac{1}{\alpha^{2}} \int_{\mathbb{R}^{3}}\left|\nabla \times \mathbf{A}_{n}\right|^{2}
\end{aligned}
$$

We consider each term separately. First, we look at the potential term, whose weak-* continuity is proved in the following lemma:

Lemma II.7 (Weak-* continuity of the potential term). Let $\gamma_{n}, \widetilde{\gamma}, \mathbf{A}_{n}$ and $\boldsymbol{\alpha}$ be the one defined in (47), (48) and (49), then we have that

$$
\lim _{n \rightarrow+\infty} \int_{\mathbb{R}^{3}} \rho_{\gamma_{n}} v=\int_{\mathbb{R}^{3}} \rho_{\widetilde{\gamma}} v
$$

Proof. Note that for every $\eta>0$, it is possible to decompose $v$ in the following way: $v=$ $v_{1}^{\eta}+v_{2}^{\eta}$, where $v_{1}^{\eta} \in L^{3 / 2}\left(\mathbb{R}^{3}\right), v_{2}^{\eta} \in L^{\infty}\left(\mathbb{R}^{3}\right)$ and $\left\|v_{2}^{\eta}\right\|_{L^{\infty}}<\eta$. In fact, $v(\mathbf{x})=-\sum_{k=1}^{K} \frac{Z_{k}}{\left|\mathbf{x}-\mathbf{R}_{k}\right|}$ and, for each $k$, we can write

$$
\frac{1}{\left|\mathbf{x}-\mathbf{R}_{k}\right|}=\underbrace{\frac{1}{\left|\mathbf{x}-\mathbf{R}_{k}\right|} \mathbb{1}_{\left\{\left|\mathbf{x}-\mathbf{R}_{k}\right| \leq 1 / \eta\right\}}}_{v_{1}^{\eta, k}}+\underbrace{\frac{1}{\left|\mathbf{x}-\mathbf{R}_{k}\right|} \mathbb{1}_{\left\{\left|\mathbf{x}-\mathbf{R}_{k}\right|>1 / \eta\right\}}}_{v_{2}^{\eta, k}} .
$$

It is easy to see that

$$
\begin{aligned}
\int_{\mathbb{R}^{3}}\left|\frac{1}{\left|\mathbf{x}-\mathbf{R}_{k}\right|} \mathbb{1}_{\left\{\left|\mathbf{x}-\mathbf{R}_{k}\right| \leq 1 / \eta\right\}}\right|^{3 / 2} d \mathbf{x} & =\int_{\left\{\left|\mathbf{x}-\mathbf{R}_{k}\right| \leq 1 / \eta\right\}}\left|\frac{1}{\left|\mathbf{x}-\mathbf{R}_{k}\right|}\right|^{3 / 2} d \mathbf{x} \\
& =2 \pi^{2} \int_{0}^{1 / \eta} \frac{1}{r^{3 / 2}} \cdot r^{2} d r=\frac{4 \pi^{2}}{3 \eta^{3 / 2}}<+\infty
\end{aligned}
$$

and that $\left\|v_{2}^{\eta, k}\right\|_{L^{\infty}}<\eta$. Define, $v_{j}^{\eta}=\sum_{k=1}^{K} Z_{k} v_{j}^{\eta, k}$ for $j=1$, 2 , then it is clear that $v_{j}^{\eta}$ for $j=1,2$ has the required properties.

Thus, for each $\eta>0$, since $v_{1}^{\eta} \in L^{3 / 2}\left(\mathbb{R}^{3}\right)$ (the dual space of $L^{3}\left(\mathbb{R}^{3}\right)$ ) and $\rho_{\gamma_{n}} \underset{n}{\rightarrow} \rho_{\widetilde{\gamma}}$ weakly in $L^{3}\left(\mathbb{R}^{3}\right)$, we obtain that

$$
\lim _{n \rightarrow+\infty} \int_{\mathbb{R}^{3}} \rho_{\gamma_{n}} v_{1}^{\eta}=\int_{\mathbb{R}^{3}} \rho_{\widetilde{\gamma}} v_{1}^{\eta}
$$


Hartree-Fock Theory with a Self-Generated Magnetic Field

On the other hand,

$$
\left|\int_{\mathbb{R}^{3}} \rho_{\gamma_{n}} v_{2}^{\eta}-\int_{\mathbb{R}^{3}} \rho_{\widetilde{\gamma}} v_{2}^{\eta}\right| \leq \int_{\mathbb{R}^{3}}\left|\rho_{\gamma_{n}}-\rho_{\widetilde{\gamma}}\right|\left|v_{2}^{\eta}\right| \leq\left\|\rho_{\gamma_{n}}-\rho_{\widetilde{\gamma}}\right\|_{L^{1}}\left\|v_{2}^{\eta}\right\|_{L^{\infty}} \leq 2 N \eta
$$

The lemma thus follows.

The next step is proving the weak-* lower semi-continuity of the direct and exchange Coulomb term:

Lemma II.8 (Weak-* lower semi-continuity of the direct and exchange Coulomb term). Let $\gamma_{n}, \widetilde{\gamma}, \mathbf{A}_{n}$ and $\boldsymbol{\alpha}$ be the one defined in (47), (48) and (49), then we have that

$\liminf _{n \rightarrow+\infty} \iint_{\mathbb{R}^{3} \times \mathbb{R}^{3}} \frac{\rho_{\gamma_{n}}(\mathbf{x}) \rho_{\gamma_{n}}(\mathbf{y})-\operatorname{Tr}_{\mathbb{C}^{2}}\left(\left|\gamma_{n}(\mathbf{x}, \mathbf{y})\right|^{2}\right)}{|\mathbf{x}-\mathbf{y}|} d \mathbf{x} d \mathbf{y} \geq \iint_{\mathbb{R}^{3} \times \mathbb{R}^{3}} \frac{\rho_{\widetilde{\gamma}}(\mathbf{x}) \rho_{\widetilde{\gamma}}(\mathbf{y})-\operatorname{Tr}_{\mathbb{C}^{2}}\left(|\widetilde{\gamma}(\mathbf{x}, \mathbf{y})|^{2}\right)}{|\mathbf{x}-\mathbf{y}|} d \mathbf{x} d \mathbf{y}$

Proof. Since $\left\{\gamma_{n}\right\}_{n \in \mathbb{N}}$ is bounded in $\mathcal{B}$, its kernel $\gamma_{n}(\mathbf{x}, \mathbf{y})$ is bounded in $H^{1}\left(\mathbb{R}^{3} \times \mathbb{R}^{3}, \operatorname{Mat}_{2 \times 2}(\mathbb{C})\right)$ and $\sqrt{\rho_{\gamma_{n}}}$ is bounded in $H^{1}\left(\mathbb{R}^{3}\right)$. Hence, up to a subsequence, we can assume that:

$$
\begin{aligned}
\rho_{\gamma_{n}} \underset{n}{\rightarrow} \rho_{\widetilde{\gamma}} & \text { in } L_{\text {loc }}^{p}\left(\mathbb{R}^{3}\right) \text { for } 1 \leq p<3 \text { and a.e. } \\
\gamma_{n}(\mathbf{x}, \mathbf{y}) \underset{n}{\rightarrow} \widetilde{\gamma}(\mathbf{x}, \mathbf{y}) & \text { in } L_{\text {loc }}^{q}\left(\mathbb{R}^{3}\right) \text { for } 2 \leq q<3 \text { and a.e. }
\end{aligned}
$$

Thus, from (27) and Fatou's lemma, (54) follows.

Given that $\nabla \times \mathbf{A}_{n} \underset{n}{\rightarrow} \nabla \times \boldsymbol{\alpha}$ weakly in $L^{2}\left(\mathbb{R}^{3}\right)$, from weakly lower semicontinuity of norms, it also follows that:

$$
\liminf _{n \rightarrow+\infty}\left\|\nabla \times \mathbf{A}_{n}\right\|_{L^{2}}^{2} \geq\|\nabla \times \boldsymbol{\alpha}\|_{L^{2}}^{2}
$$

Finally, note that if we prove that:

$$
\sigma \cdot\left(i \nabla-\mathbf{A}_{n}\right) \gamma_{n}\left(i \nabla-\mathbf{A}_{n}\right) \cdot \sigma \underset{n}{\stackrel{*}{\rightarrow}} \sigma \cdot(i \nabla-\boldsymbol{\alpha}) \widetilde{\gamma}(i \nabla-\boldsymbol{\alpha}) \cdot \sigma
$$

weakly-* in $\mathcal{S}_{1}$, the space of trace-class operators on $L^{2}\left(\mathbb{R}^{3}, \mathbb{C}^{2}\right)$, then:

$$
\liminf _{n \rightarrow+\infty} \frac{1}{2} \operatorname{Tr}\left(\sigma \cdot\left(i \nabla-\mathbf{A}_{n}\right) \gamma_{n}\left(i \nabla-\mathbf{A}_{n}\right) \cdot \sigma\right) \geq \frac{1}{2} \operatorname{Tr}(\sigma \cdot(i \nabla-\boldsymbol{\alpha}) \widetilde{\gamma}(i \nabla-\boldsymbol{\alpha}) \cdot \sigma)
$$

will follow from Fatou's lemma for series.

The weak-* convergence of the kinetic energy term is treated in the following lemma: 
Hartree-Fock Theory with a Self-Generated Magnetic Field

Lemma II.9 (Weak-* convergence of the kinetic energy term). For $n$ going to infinity,

$$
\sigma \cdot\left(i \nabla-\mathbf{A}_{n}\right) \gamma_{n}\left(i \nabla-\mathbf{A}_{n}\right) \cdot \sigma \underset{n}{\stackrel{*}{\rightarrow}} \sigma \cdot(i \nabla-\boldsymbol{\alpha}) \widetilde{\gamma}(i \nabla-\boldsymbol{\alpha}) \cdot \sigma
$$

weakly-* in $\mathcal{S}_{1}$.

Proof. First note that it is sufficient to prove:

$$
\left(i \nabla-\mathbf{A}_{n}\right) \gamma_{n}\left(i \nabla-\mathbf{A}_{n}\right) \underset{n}{\stackrel{*}{\rightarrow}}(i \nabla-\boldsymbol{\alpha}) \widetilde{\gamma}(i \nabla-\boldsymbol{\alpha})
$$

weakly-* in $\mathcal{S}_{1}$. In fact, the Pauli operator $\sigma$ does not compromise the weak-* convergence since it has the only effect of mixing the order of the components of $\left(i \nabla-\mathbf{A}_{n}\right) \gamma_{n}\left(i \nabla-\mathbf{A}_{n}\right)$. Thus, we shall prove (61) directly. In order to do that, for any $n \in \mathbb{N}$, we define the auxiliary positive operators:

$$
\begin{aligned}
\tau_{n} & =\left(-i+\left(i \nabla-\mathbf{A}_{n}\right)\right) \gamma_{n}\left(i+\left(i \nabla-\mathbf{A}_{n}\right)\right) \\
& =\gamma_{n}-i \gamma_{n}\left(i \nabla-\mathbf{A}_{n}\right)+i\left(i \nabla-\mathbf{A}_{n}\right) \gamma_{n}+\left(i \nabla-\mathbf{A}_{n}\right) \gamma_{n}\left(i \nabla-\mathbf{A}_{n}\right)
\end{aligned}
$$

As the following lemma proves, these operators are uniformly bounded in $\mathcal{S}_{1}$ :

Lemma II.10 (Boundedness in $\left.\mathcal{S}_{1}\right)$. The operators $\left\{\tau_{n}\right\}_{n \in \mathbb{N}}$ can be extended to $L^{2}\left(\mathbb{R}^{3}, \mathbb{C}^{2}\right)$ and are uniformly bounded in $\mathcal{S}_{1}$.

Proof. Note that, since $\left(\gamma_{n}, \mathbf{A}_{n}\right) \in \mathcal{C}$, then, in particular, $\gamma_{n}$ is a positive trace class operator defined on $L^{2}\left(\mathbb{R}^{3}, \mathbb{C}^{2}\right)$ and $\left\|\gamma_{n}\right\|_{\mathcal{S}_{1}}=\operatorname{Tr}\left(\gamma_{n}\right)=N$, which means that $\left\{\gamma_{n}\right\}_{n \in \mathbb{N}}$ is uniformly bounded in $\mathcal{S}_{1}$.

On the other hand, the operators $\gamma_{n}\left(i \nabla-\mathbf{A}_{n}\right)$ and $\left(i \nabla-\mathbf{A}_{n}\right) \gamma_{n}$ are defined in $\mathcal{D}\left(i \nabla-\mathbf{A}_{n}\right)$, the domain of $\left(i \nabla-\mathbf{A}_{n}\right)$, but can be extended to $L^{2}\left(\mathbb{R}^{3}, \mathbb{C}^{2}\right)$, where they are bounded and adjoint of each others. Moreover, they are in $\mathcal{S}_{1}$ and the sequence $\left\{\operatorname{Tr}\left(\gamma_{n}\left(i \nabla-\mathbf{A}_{n}\right)\right)=\right.$ $\left.\operatorname{Tr}\left(\left(i \nabla-\mathbf{A}_{n}\right) \gamma_{n}\right)\right\}_{n \in \mathbb{N}}$ is uniformly bounded.

In fact, let $\psi$ be in $\mathcal{D}\left(i \nabla-\mathbf{A}_{n}\right)$ and let $\gamma_{n}$ have decomposition $\gamma_{n}=\sum_{k=1}^{+\infty} \lambda_{k}^{n} \phi_{k}^{n} \otimes \phi_{k}^{n}$. Thus,

$$
\gamma_{n}\left(i \nabla-\mathbf{A}_{n}\right) \psi=\sum_{k=1}^{+\infty} \lambda_{k}^{n}\left(\phi_{k}^{n},\left(i \nabla-\mathbf{A}_{n}\right) \psi\right)_{L^{2}\left(\mathbb{R}^{3}, \mathbb{C}^{2}\right)} \phi_{k}^{n}
$$

We want to show that $\phi_{k}^{n} \in \mathcal{D}\left(i \nabla-\mathbf{A}_{n}\right)$. In fact, there exists a constant $D_{1}$ such that for every $\psi \in \mathcal{D}\left(i \nabla-\mathbf{A}_{n}\right),\left(\phi_{k}^{n},\left(i \nabla-\mathbf{A}_{n}\right) \psi\right)_{L^{2}\left(\mathbb{R}^{3}, \mathbb{C}^{2}\right)} \leq D_{1}\|\psi\|_{L^{2}\left(\mathbb{R}^{3}, \mathbb{C}^{2}\right)}$. This follows from the 
Hartree-Fock Theory with a Self-Generated Magnetic Field

following inequalities (for clarity reasons, we omit the subscript $L^{2}\left(\mathbb{R}^{3}, \mathbb{C}^{2}\right)$ when the inner product or norm we are using is clear):

$$
\begin{aligned}
\lambda_{k}^{n}\left(\phi_{k}^{n},\left(i \nabla-\mathbf{A}_{n}\right) \psi\right)^{2} & \leq \sum_{k=1}^{+\infty} \lambda_{k}^{n}\left(\phi_{k}^{n},\left(i \nabla-\mathbf{A}_{n}\right) \psi\right)^{2} \\
& =\left(\left(i \nabla-\mathbf{A}_{n}\right) \psi, \gamma_{n}\left(i \nabla-\mathbf{A}_{n}\right) \psi\right) \\
& =\left(\psi,\left(i \nabla-\mathbf{A}_{n}\right) \gamma_{n}\left(i \nabla-\mathbf{A}_{n}\right) \psi\right) \\
& \leq\left\|\left(i \nabla-\mathbf{A}_{n}\right) \gamma_{n}\left(i \nabla-\mathbf{A}_{n}\right)\right\|\|\psi\|_{L^{2}}^{2}
\end{aligned}
$$

where the first inequality follows from $\lambda_{k}^{n}>0$ and the last one from the boundedness of $\left(i \nabla-\mathbf{A}_{n}\right) \gamma_{n}\left(i \nabla-\mathbf{A}_{n}\right)$ in $\mathcal{S}_{1}$. In this paper, given an operator $T$, we denote its operator norm by $\|T\|$ and its adjoint by $T^{*}$.

It follows from (65) that the functional:

$$
\begin{aligned}
\mathcal{D}(i \nabla-\mathbf{A}) & \longrightarrow \mathbb{C} \\
\psi & \longrightarrow\left(\phi_{k}^{n},\left(i \nabla-\mathbf{A}_{n}\right) \psi\right)
\end{aligned}
$$

is linear and bounded and can thus be extended to $L^{2}\left(\mathbb{R}^{3}, \mathbb{C}^{2}\right)$. Hence, for each $k, \phi_{k}^{n} \in$ $\mathcal{D}\left(i \nabla-\mathbf{A}_{n}\right)^{*}=\mathcal{D}\left(i \nabla-\mathbf{A}_{n}\right)$, since $\left(i \nabla-\mathbf{A}_{n}\right)$ is self-adjoint. Thus, (64) becomes

$$
\gamma_{n}\left(i \nabla-\mathbf{A}_{n}\right) \psi=\sum_{k=1}^{+\infty} \lambda_{k}^{n} \phi_{k}^{n}\left(\left(i \nabla-\mathbf{A}_{n}\right) \phi_{k}^{n}, \psi\right)
$$

and

$$
\begin{aligned}
\left\|\gamma_{n}\left(i \nabla-\mathbf{A}_{n}\right) \psi\right\|_{L^{2}}^{2} & =\sum_{k=1}^{+\infty}\left(\lambda_{k}^{n}\right)^{2}\left|\left(\left(i \nabla-\mathbf{A}_{n}\right) \phi_{k}^{n}, \psi\right)\right|^{2} \\
& \leq \sum_{k=1}^{+\infty}\left(\lambda_{k}^{n}\right)^{2}\left\|\left(i \nabla-\mathbf{A}_{n}\right) \phi_{k}^{n}\right\|_{L^{2}}^{2}\|\psi\|_{L^{2}}^{2} \\
& \leq \sum_{k=1}^{+\infty}\left(\lambda_{k}^{n}\right)\left\|\left(i \nabla-\mathbf{A}_{n}\right) \phi_{k}^{n}\right\|_{L^{2}}^{2}\|\psi\|_{L^{2}}^{2} \\
& =\operatorname{Tr}\left(\left(i \nabla-\mathbf{A}_{n}\right) \gamma_{n}\left(i \nabla-\mathbf{A}_{n}\right)\right)\|\psi\|_{L^{2}}^{2} \\
& \leq D_{2}\|\psi\|_{L^{2}}^{2},
\end{aligned}
$$

given that $\left\{\left(i \nabla-\mathbf{A}_{n}\right) \gamma_{n}\left(i \nabla-\mathbf{A}_{n}\right)\right\}_{n \in \mathbb{N}}$ is uniformly bounded in $\mathcal{S}_{1}$.

As a consequence, $\gamma_{n}\left(i \nabla-\mathbf{A}_{n}\right)$ can be extended to a bounded operator defined on the entire 
Hartree-Fock Theory with a Self-Generated Magnetic Field

space $L^{2}\left(\mathbb{R}^{3}, \mathbb{C}^{2}\right)$.

Also, $\left(i \nabla-\mathbf{A}_{n}\right) \gamma_{n}=\left(\gamma_{n}\left(i \nabla-\mathbf{A}_{n}\right)\right)^{*}$. In fact, for every $\psi, \theta \in L^{2}\left(\mathbb{R}^{3}, \mathbb{C}^{2}\right)$

$$
\begin{aligned}
\left(\left(i \nabla-\mathbf{A}_{n}\right) \gamma_{n} \psi, \theta\right) & =\sum_{k=1}^{+\infty} \lambda_{k}^{n}\left(\phi_{k}^{n}, \theta\right)\left(\left(i \nabla-\mathbf{A}_{n}\right) \phi_{k}^{n}, \psi\right) \\
& =\left(\psi,\left(i \nabla-\mathbf{A}_{n}\right) \gamma_{n} \theta\right) .
\end{aligned}
$$

Finally, note that:

$$
\left(\gamma_{n}^{1 / 2}\left(i \nabla-\mathbf{A}_{n}\right)\right)^{*}\left(\gamma_{n}^{1 / 2}\left(i \nabla-\mathbf{A}_{n}\right)\right)=\left(i \nabla-\mathbf{A}_{n}\right) \gamma_{n}\left(i \nabla-\mathbf{A}_{n}\right) \in \mathcal{S}_{1}
$$

which implies that $\gamma_{n}^{1 / 2}\left(i \nabla-\mathbf{A}_{n}\right) \in \mathcal{S}_{2}$, where $\mathcal{S}_{2}$ is the space of Hilbert-Schmidt operators on $L^{2}\left(\mathbb{R}^{3}, \mathbb{C}^{2}\right)$. Thus, $\gamma_{n}\left(i \nabla-\mathbf{A}_{n}\right)=\underbrace{\left(\gamma_{n}^{1 / 2}\right)}_{\in \mathcal{S}_{2}} \underbrace{\left(\gamma_{n}^{1 / 2}\left(i \nabla-\mathbf{A}_{n}\right)\right)}_{\in \mathcal{S}_{2}} \in \mathcal{S}_{1}$ and clearly also $\left(i \nabla-\mathbf{A}_{n}\right) \gamma_{n}=$ $\left(\gamma_{n}\left(i \nabla-\mathbf{A}_{n}\right)\right)^{*} \in \mathcal{S}_{1}$. Moreover:

$$
\begin{aligned}
\left(\operatorname{Tr}\left(\left(i \nabla-\mathbf{A}_{n}\right) \gamma_{n}\right)\right)^{2}=\left(\operatorname{Tr}\left(\gamma_{n}\left(i \nabla-\mathbf{A}_{n}\right)\right)\right)^{2} & =\operatorname{Tr}\left(\gamma_{n}^{1 / 2} \gamma_{n}^{1 / 2}\left(i \nabla-\mathbf{A}_{n}\right)\right) \\
& \leq\left\|\gamma_{n}\right\|_{\mathcal{S}_{2}}^{2}\left\|\gamma_{n}^{1 / 2}\left(i \nabla-\mathbf{A}_{n}\right)\right\|_{\mathcal{S}_{2}}^{2} \\
& =\operatorname{Tr}\left(\gamma_{n}\right) \operatorname{Tr}\left(\left(i \nabla-\mathbf{A}_{n}\right) \gamma_{n}\left(i \nabla-\mathbf{A}_{n}\right)\right)
\end{aligned}
$$

which, as we have seen before, are both uniformly bounded. As a consequence, $\operatorname{Tr}\left(\gamma_{n}(i \nabla-\right.$ $\left.\left.\mathbf{A}_{n}\right)\right)$ and $\operatorname{Tr}\left(\left(i \nabla-\mathbf{A}_{n}\right) \gamma_{n}\right)$ are both uniformly bounded, as claimed.

In the same way, $\left(i \nabla-\mathbf{A}_{n}\right) \gamma_{n}\left(i \nabla-\mathbf{A}_{n}\right)$ is a positive operator that can be extended to $L^{2}\left(\mathbb{R}^{3}, \mathbb{C}^{2}\right)$ and is uniformly bounded in $\mathcal{S}_{1}$.

In conclusion, the operators $\tau_{n}$ are positive, can be extended to $L^{2}\left(\mathbb{R}^{3}, \mathbb{C}^{2}\right)$ and given that

$$
\operatorname{Tr}\left(\tau_{n}\right)=\operatorname{Tr}\left(\gamma_{n}\right)-i \operatorname{Tr}\left(\gamma_{n}\left(i \nabla-\mathbf{A}_{n}\right)\right)+i \operatorname{Tr}\left(\left(i \nabla-\mathbf{A}_{n}\right) \gamma_{n}\right)+\operatorname{Tr}\left(\left(i \nabla-\mathbf{A}_{n}\right) \gamma_{n}\left(i \nabla-\mathbf{A}_{n}\right)\right)
$$

is a sum of terms which are all uniformly bounded in $\mathcal{S}_{1},\left\{\tau_{n}\right\}_{n \in \mathbb{N}}$ is uniformly bounded in $\mathcal{S}_{1}$

Consider the following operators:

$$
\begin{aligned}
R_{n} & =\left(-i+\left(i \nabla-\mathbf{A}_{n}\right)\right)^{-1} \\
R & =(-i+(i \nabla-\boldsymbol{\alpha}))^{-1}
\end{aligned}
$$

Standard results on self-adjoint operators (see Chapter $\mathrm{X}$ of Ref. 30) imply that $R_{n}$ is a bounded invertible operator and that:

$$
\left\|R_{n}\right\| \leq \operatorname{dist}\left(-\mathrm{i}, \operatorname{spec}\left(\left(\mathrm{i} \nabla-\mathbf{A}_{\mathrm{n}}\right)\right)\right)^{-1} \leq \widetilde{\mathrm{k}}
$$


Hartree-Fock Theory with a Self-Generated Magnetic Field

where $\widetilde{k}$ is a constant, $\operatorname{dist}(\cdot, \cdot)$ is the Euclidean distance and $\operatorname{spec}\left(\mathrm{i} \nabla-\mathbf{A}_{\mathrm{n}}\right)$ is the spectrum of $\left(i \nabla-\mathbf{A}_{n}\right)$. In other words, $\left\{R_{n}\right\}_{n \in \mathbb{N}}$ is uniformly bounded in $\mathcal{S}\left(L^{2}\left(\mathbb{R}^{3}, \mathbb{C}^{2}\right)\right)$. Clearly, the same holds for its adjoint, $R_{n}^{*}=\left(i+\left(i \nabla-\mathbf{A}_{n}\right)\right)^{-1}$, and for $R$ and its adjoint, $R^{*}=$ $(i+(i \nabla-\boldsymbol{\alpha}))^{-1}$. By definition, it holds that:

$$
\gamma_{n}=R_{n} \tau_{n} R_{n}^{*}
$$

Note that Lemma II.10 implies that there exists $\widetilde{\tau} \in \mathcal{S}_{1}$ such that $\tau_{n} \underset{n}{\stackrel{*}{\rightarrow}} \widetilde{\tau}$ weakly-* in $\mathcal{S}_{1}$. Also, in (49), we proved that there exists $\widetilde{\gamma} \in \mathcal{B}$ such that $\gamma_{n} \underset{n}{*} \widetilde{\gamma}$ weakly-* in $\mathcal{B}$.

To prove Lemma II.9, we need to identify the weak- $*$ limit in $\mathcal{B}$ of $\left(i \nabla-\mathbf{A}_{n}\right) \gamma_{n}\left(i \nabla-\mathbf{A}_{n}\right)$. This is equivalent to identifying, for every $\phi, \psi \in C_{0}^{\infty}\left(\mathbb{R}^{3}, \mathbb{C}^{2}\right)$, smooth functions with compact support, the limit of $\left(\phi,\left(i \nabla-\mathbf{A}_{n}\right) \gamma_{n}\left(i \nabla-\mathbf{A}_{n}\right) \psi\right)$. Let us write:

$$
\left(\phi,\left(i \nabla-\mathbf{A}_{n}\right) \gamma_{n}\left(i \nabla-\mathbf{A}_{n}\right) \psi\right)=\left(\phi, \tau_{n} \psi\right)-\left(\phi, \gamma_{n} \psi\right)+i\left(\phi, \gamma_{n}\left(i \nabla-\mathbf{A}_{n}\right) \psi\right)-i\left(\phi,\left(i \nabla-\mathbf{A}_{n}\right) \gamma_{n} \psi\right)
$$

In the following lemma, we identify each limit in the right hand side of (69):

Lemma II.11. Given any $\phi, \psi \in C_{0}^{\infty}\left(\mathbb{R}^{3}, \mathbb{C}^{2}\right)$, as $n$ goes to infinity, the following limits are obtained:

$$
\begin{array}{r}
\left(\phi, \tau_{n} \psi\right) \underset{n}{\rightarrow}\left(\phi, R^{-1} \widetilde{\gamma}\left(R^{-1}\right)^{*} \psi\right) \\
\left(\phi, \gamma_{n} \psi\right) \underset{n}{\rightarrow}(\phi, \widetilde{\gamma} \psi) \\
\left(\phi, \gamma_{n}\left(i \nabla-\mathbf{A}_{n}\right) \psi\right) \underset{n}{\rightarrow}(\phi,(R \widetilde{\tau}-i \widetilde{\gamma}) \psi) \\
\left(\phi,\left(i \nabla-\mathbf{A}_{n}\right) \gamma_{n} \psi\right) \underset{n}{\rightarrow}\left(\phi,\left(\widetilde{\tau} R^{*}+i \widetilde{\gamma}\right) \psi\right)
\end{array}
$$

Proof. Since $\tau_{n} \underset{n}{\stackrel{*}{*}} \widetilde{\tau}$ weakly-* in $\mathcal{S}_{1}$, then clearly $\left(\phi, \tau_{n} \psi\right) \underset{n}{\rightarrow}(\phi, \widetilde{\tau} \psi)$.

We want to show that:

$$
\widetilde{\tau}=R^{-1} \widetilde{\gamma}\left(R^{-1}\right)^{*}
$$

First, note that:

$$
\begin{aligned}
\left(\phi, \gamma_{n} \psi\right) & =\left(\phi, R_{n} \tau_{n} R_{n}^{*} \psi\right) \\
& =\left(R_{n}^{*} \phi, \tau_{n} R_{n}^{*} \psi\right) \\
& =\left(R_{n}^{*} \phi-R^{*} \phi, \tau_{n} R_{n}^{*} \psi\right)+\left(R^{*} \phi, \tau_{n}\left(R_{n}^{*} \psi-R^{*} \psi\right)\right)+\left(R^{*} \phi, \tau_{n} R^{*} \psi\right)
\end{aligned}
$$


Hartree-Fock Theory with a Self-Generated Magnetic Field

where (76) follows since $R_{n}$ is bounded. Let us consider the limit for $n$ going to infinity of (77). Note that:

$$
0 \leq\left|\left(R_{n}^{*} \phi-R^{*} \phi, \tau_{n} R_{n}^{*} \psi\right)\right| \leq\left\|R_{n}^{*} \phi-R^{*} \phi\right\|_{L^{2}}\left\|\tau_{n}\right\|\left\|R_{n}^{*}\right\|\|\psi\|_{L^{2}} \leq D_{3}\left\|R_{n}^{*} \phi-R^{*} \phi\right\|_{L^{2}}\|\psi\|_{L^{2}}
$$

where $D_{3}$ is constant. The inequality above follows from the fact that both $\left\{\tau_{n}\right\}_{n \in \mathbb{N}}$ and $\left\{R_{n}^{*}\right\}_{n \in \mathbb{N}}$ are uniformly bounded in $\mathcal{S}\left(L^{2}\left(\mathbb{R}^{3}, \mathbb{C}^{2}\right)\right)$.

In addition, we have strong convergence in $L^{2}\left(\mathbb{R}^{3}, \mathbb{C}^{2}\right)$ of $R_{n}^{*} \underset{n}{\rightarrow} R^{*}$, as showed by the following lemma:

Lemma II.12 (Strong convergence of $R_{n}$ ). For $n$ going to infinity, $R_{n} \rightarrow R$ strongly in $L^{2}\left(\mathbb{R}^{3}, \mathbb{C}^{2}\right)$.

Proof. Note that

$$
\begin{aligned}
0 & \leq\left\|R_{n} \phi-R \phi\right\|_{L^{2}}=\left\|R_{n}\left(R_{n}^{-1}-R^{-1}\right) R \phi\right\|_{L^{2}} \leq\left\|R_{n}\right\|\left\|\left(R_{n}^{-1}-R^{-1}\right) R \phi\right\|_{L^{2}} \\
& \leq D_{4}\left\|\left(\mathbf{A}_{n}-\boldsymbol{\alpha}\right) R \phi\right\|_{L^{2}}
\end{aligned}
$$

where $D_{4}$ is constant and (80) follows since $\left\{R_{n}\right\}_{n \in \mathbb{N}}$ is uniformly bounded in $\mathcal{S}\left(L^{2}\left(\mathbb{R}^{3}, \mathbb{C}^{2}\right)\right)$. Moreover, for every $\phi \in L^{2}\left(\mathbb{R}^{3}, \mathbb{C}^{2}\right), R \phi \in H_{\boldsymbol{\alpha}}^{1}\left(\mathbb{R}^{3}, \mathbb{C}^{2}\right)$, that is, $R \phi \in L^{2}\left(\mathbb{R}^{3}, \mathbb{C}\right)$ and $(i \nabla-$ $\boldsymbol{\alpha}) R \phi \in L^{2}\left(\mathbb{R}^{3}, \operatorname{Mat}_{3 \times 2}(\mathbb{C})\right)$. As a consequence of the diamagnetic inequality $|R \phi| \in H^{1}\left(\mathbb{R}^{3}\right)$. Thus, if we prove that for every $\theta \in H^{1}\left(\mathbb{R}^{3}, \mathbb{C}^{2}\right),\left\|\left(\mathbf{A}_{n}-\boldsymbol{\alpha}\right) \theta\right\|_{L^{2}} \underset{n}{\rightarrow} 0$, then we can conclude that $\left\|R_{n} \phi-R \phi\right\|_{L^{2}} \underset{n}{\rightarrow} 0$.

First, consider any $\theta \in C_{0}^{\infty}\left(\mathbb{R}^{3}, \mathbb{C}^{2}\right)$, then:

$$
\begin{aligned}
0 & \leq\left\|\left(\mathbf{A}_{n}-\boldsymbol{\alpha}\right) \theta\right\|_{L^{2}}=\int_{\operatorname{supp}(\theta)}\left|\mathbf{A}_{n}-\boldsymbol{\alpha}\right|^{2}|\theta|^{2} \\
& \leq\left(\int_{\operatorname{supp}(\theta)}\left|\mathbf{A}_{n}-\boldsymbol{\alpha}\right|^{4}\right)^{1 / 2}\left(\int_{\operatorname{supp}(\theta)}|\theta|^{4}\right)^{1 / 2} .
\end{aligned}
$$

Since $\left\{\mathbf{A}_{n}\right\}_{n \in \mathbb{N}}$ is uniformly bounded in $L^{6}\left(\mathbb{R}^{3}\right)$ and $\mathbf{A}_{n} \underset{n}{\rightarrow} \boldsymbol{\alpha}$ weakly in $L^{6}\left(\mathbb{R}^{3}\right)$, RellichKondrachov theorem implies that $A_{n} \underset{n}{\rightarrow} \boldsymbol{\alpha}$ in $L_{\text {loc }}^{4}\left(\mathbb{R}^{3}\right)$. Hence, (82) implies that for every $\theta \in C_{0}^{\infty}\left(\mathbb{R}^{3}, \mathbb{C}^{2}\right),\left\|\left(\mathbf{A}_{n}-\boldsymbol{\alpha}\right) \theta\right\|_{L^{2}} \underset{n}{\rightarrow} 0$. By density of $C_{0}^{\infty}\left(\mathbb{R}^{3}, \mathbb{C}^{2}\right)$ in $H^{1}\left(\mathbb{R}^{3}, \mathbb{C}^{2}\right)$, the same result follows for any $\theta \in H^{1}\left(\mathbb{R}^{3}, \mathbb{C}^{2}\right)$, as claimed.

It is clear that the same result of Lemma II.12 holds for $R_{n}^{*}$ and $R^{*}$. Hence, Lemma II.12 and (78) imply that $\left(R_{n}^{*} \phi-R^{*} \phi, \tau_{n} R_{n}^{*} \psi\right) \underset{n}{\rightarrow} 0$ as $\mathrm{n}$ goes to infinity. 
In the same way, it is possible to prove that $\left(R^{*} \phi, \tau_{n}\left(R_{n}^{*} \psi-R^{*} \psi\right)\right) \underset{n}{\rightarrow} 0$ and, since $\tau_{n} \underset{n}{\stackrel{*}{*}} \widetilde{\tau}$ weakly-* in $\mathcal{S}_{1}$, then we also have that $\left(R^{*} \phi, \tau_{n} R^{*} \psi\right) \underset{n}{\rightarrow}\left(R^{*} \phi, \widetilde{\tau} R^{*} \psi\right)$.

Now, recall that $\gamma_{n} \underset{n}{\stackrel{*}{*}} \widetilde{\gamma}$ weakly-* in $\mathcal{B}$ and take the limit in (77) for $\mathrm{n}$ going to infinity. We obtain that for every $\phi, \psi \in C_{0}^{\infty}\left(\mathbb{R}^{3}, \mathbb{C}^{2}\right)$ :

$$
(\phi, \widetilde{\gamma} \psi)=\left(R^{*} \phi, \widetilde{\tau} R^{*} \psi\right)=\left(\phi, R \widetilde{\tau} R^{*} \psi\right)
$$

It follows that $\widetilde{\gamma}=R \widetilde{\tau} R^{*}$ and $\widetilde{\tau}=R^{-1} \widetilde{\gamma}\left(R^{-1}\right)^{*}$, which proves (74) and thus (70).

Moreover, since $\gamma_{n} \underset{n}{\stackrel{*}{*}} \widetilde{\gamma}$ weakly-* in $\mathcal{B}$, then clearly (71) follows.

In addition, note that the following identities hold:

$$
\begin{aligned}
\left(\phi, \gamma_{n}\left(i \nabla-\mathbf{A}_{n}\right) \psi\right) & =\left(\phi, \gamma_{n}\left(R_{n}^{-1}\right)^{*} \psi\right)-i\left(\phi, \gamma_{n} \psi\right) \\
& =\left(\phi, R_{n} R_{n}^{-1} \gamma_{n}\left(R_{n}^{-1}\right)^{*} \psi\right)-i\left(\phi, \gamma_{n} \psi\right) \\
& =\left(\phi, R_{n} \tau_{n} \psi\right)-i\left(\phi, \gamma_{n} \psi\right) \\
& =\left(R_{n}^{*} \phi, \tau_{n} \psi\right)-i\left(\phi, \gamma_{n} \psi\right) \\
& \underset{n}{\rightarrow}\left(R^{*} \phi, \widetilde{\tau} \psi\right)-i(\phi, \widetilde{\gamma} \psi),
\end{aligned}
$$

where (86) holds since $R_{n}$ is bounded and (87) follows from Lemma II.12 and the fact that $\tau_{n} \underset{n}{\stackrel{*}{*}} \widetilde{\tau}$ weakly-* in $\mathcal{S}_{1}$. Thus, (72) follows and, in the same way, it is possible to prove $(73)$.

Now, if we take the limit for $n$ going to infinity on both sides of (69), Lemma II.11 implies that for every $\phi, \psi \in C_{0}^{\infty}\left(\mathbb{R}^{3}, \mathbb{C}^{2}\right)$ :

$$
\begin{gathered}
\left(\phi,\left(i \nabla-\mathbf{A}_{n}\right) \gamma_{n}\left(i \nabla-\mathbf{A}_{n}\right) \psi\right) \underset{n}{\rightarrow}(\phi,(\widetilde{\tau}-\widetilde{\gamma}+i R \widetilde{\tau}+\widetilde{\gamma}-i \widetilde{\tau} S+\widetilde{\gamma}) \psi) \\
=(\phi,(\widetilde{\tau}+i R \widetilde{\tau}-i \widetilde{\tau} S+\widetilde{\gamma}) \psi)
\end{gathered}
$$

which means that $\left(i \nabla-\mathbf{A}_{n}\right) \gamma_{n}\left(i \nabla-\mathbf{A}_{n}\right) \underset{n}{\stackrel{*}{*}} \widetilde{\tau}+i R \widetilde{\tau}-i \widetilde{\tau} S+\widetilde{\gamma}$ weakly-* in $\mathcal{S}_{1}$. If we substitute $\widetilde{\tau}=R^{-1} \widetilde{\gamma} S^{-1}$, we obtain $\left(i \nabla-\mathbf{A}_{n}\right) \gamma_{n}\left(i \nabla-\mathbf{A}_{n}\right) \underset{n}{\stackrel{*}{*}}(i \nabla-\boldsymbol{\alpha}) \widetilde{\gamma}(i \nabla-\boldsymbol{\alpha})$ weakly-* in $\mathcal{S}_{1}$, as claimed.

From (51), (54), (57) and (59), we deduce that the energy is weakly-* lower semicontinuous and the proof of Lemma II.6 is thus concluded.

We can combine all the previous results in the following theorem: 
Hartree-Fock Theory with a Self-Generated Magnetic Field

Theorem II.13. If $Z_{k} \alpha^{2} \leq 0.052$ for all $k=1, \ldots, K$ and $N \leq Z=\sum_{k=1}^{K} Z_{k}$, the functional $\mathcal{E}_{\mathrm{SMHF}}$ has a minimizer in $\mathcal{C}^{\mathrm{R}}$.

Proof. Given Lemma II.1, (47), (48), (49), Lemma II.6 and the fact that $\left(\gamma_{n}, \mathbf{A}_{n}\right)_{n \in \mathbb{N}}$ is a minimizing sequence for $(20)$, we have that

$$
E_{\mathrm{SMHF}}^{\mathrm{R}}=\liminf _{n \rightarrow+\infty} \mathcal{E}_{\mathrm{SMHF}}\left(\gamma_{n}, \mathbf{A}_{n}\right) \geq \mathcal{E}_{\mathrm{SMHF}}(\widetilde{\gamma}, \boldsymbol{\alpha})
$$

Since $(\widetilde{\gamma}, \boldsymbol{\alpha}) \in \mathcal{C}^{\mathrm{R}}$, it follows that

$$
\mathcal{E}_{\mathrm{SMHF}}(\widetilde{\gamma}, \boldsymbol{\alpha}) \geq E_{\mathrm{SMHF}}^{\mathrm{R}}
$$

Thus, $\mathcal{E}_{\mathrm{SMHF}}(\widetilde{\gamma}, \boldsymbol{\alpha})=E_{\mathrm{SMHF}}^{\mathrm{R}}=E_{\mathrm{SMHF}}$ and $(\widetilde{\gamma}, \boldsymbol{\alpha})$ is a minimizer to the relaxed problem.

To complete the proof of Theorem I.1, we need to show that there is a minimizer in $\mathcal{C}$. Clearly, it is sufficient to prove that $\operatorname{Tr}(\widetilde{\gamma})=\mathrm{N}$. We first need to make an initial remark:

Remark II.14. We can characterize $\widetilde{\gamma}$ in the following way:

$$
\widetilde{\gamma} \in \operatorname{argmin}\left\{\operatorname{Tr}\left(H_{\widetilde{\gamma}} \gamma\right): \gamma \in \mathcal{D}^{\mathrm{R}}\right\}
$$

where

$$
H_{\widetilde{\gamma}}=-\frac{1}{2}|\sigma \cdot(i \nabla-\boldsymbol{\alpha})|^{2}+\left(v+\left(\rho_{\widetilde{\gamma}} * \frac{1}{|x|}\right)\right) \mathbb{I}_{2 \times 2}-K_{\widetilde{\gamma}}
$$

In the above, $\mathbb{I}_{2 \times 2}$ is the $2 \times 2$ identity matrix and $K_{\widetilde{\gamma}}$ is the exchange operator define by the $2 \times 2$ matrix valued integral kernel $K_{\widetilde{\gamma}}(\mathbf{x}, \mathbf{y})=\frac{\widetilde{\gamma}(\mathbf{x}, \mathbf{y})}{|\mathbf{x}-\mathbf{y}|}$.

Proof. For every $\gamma \in \mathcal{D}^{\mathrm{R}}$, since $(\widetilde{\gamma}, \boldsymbol{\alpha})$ is a minimizer for $\mathcal{E}_{\mathrm{SMHF}}$ in $\mathcal{C}^{R}$, we have that $\mathcal{E}_{\mathrm{SMHF}}(\gamma, \boldsymbol{\alpha}) \geq \mathcal{E}_{\mathrm{SMHF}}(\tilde{\gamma}, \boldsymbol{\alpha})$. Therefore, $\left.\frac{d}{d t}\left(\mathcal{E}_{\mathrm{SMHF}}((1-t) \widetilde{\gamma}+t \gamma, \boldsymbol{\alpha})\right)\right|_{t=0} \geq 0$, which is equivalent to $\operatorname{Tr}\left(H_{\widetilde{\gamma}} \gamma\right) \geq \operatorname{Tr}\left(H_{\widetilde{\gamma}} \widetilde{\gamma}\right)$. In other words, $\widetilde{\gamma} \in \operatorname{argmin}\left\{\operatorname{Tr}\left(\mathrm{H}_{\widetilde{\gamma}} \gamma\right): \gamma \in \mathcal{D}^{\mathrm{R}}\right\}$, as claimed.

In addition, we need the following Lemma whose proof we postpone to the end. This Lemma extends a classical result by Lions (see Ref. 10) to the magnetic Hamiltonian.

Lemma II.15. Let $\mu$ be a bounded non negative measure on $\mathbb{R}^{3}$ s.t. $\mu\left(\mathbb{R}^{3}\right)<Z$. The Hamiltonian

$$
H_{1}=-\frac{1}{2}|\sigma \cdot(i \nabla-\boldsymbol{\alpha})|^{2}+\left(v+\left(\mu * \frac{1}{|\mathbf{x}|}\right)\right) \mathbb{I}_{2 \times 2}
$$

has an increasing sequence of negative eigenvalues converging to 0. 
Hartree-Fock Theory with a Self-Generated Magnetic Field

Let $(\widetilde{\gamma}, \boldsymbol{\alpha})$ be the minimizer for $\mathcal{C}^{R}$, with decomposition

$$
\widetilde{\gamma}=\sum_{k=1}^{+\infty} \lambda_{k} \phi_{k} \otimes \phi_{k}, \quad 0<\lambda_{k} \leq 1,
$$

(since they do not contribute to it, we are excluding from the sum all the terms with $\lambda_{k}=0$ ). First, note that $\lambda_{k}$ and $\phi_{k}$ have the following property:

Proposition II.16. If $\lambda_{k}>0$, then $\left(H_{\widetilde{\gamma}} \phi_{k}, \phi_{k}\right) \leq 0$.

Proof. Assume there exists $k^{*}$ such that $\lambda_{k^{*}} \neq 0$ but $\left(H_{\widetilde{\gamma}} \phi_{k^{*}}, \phi_{k^{*}}\right)>0$.

Define $\gamma^{*}=\widetilde{\gamma}-\lambda_{k^{*}} \phi_{k^{*}} \otimes \phi_{k^{*}}$. Note that $\operatorname{Tr}\left(\gamma^{*}\right)=\sum_{k=1, k \neq k^{*}}^{+\infty} \lambda_{k}<\operatorname{Tr}(\widetilde{\gamma}) \leq N$ and that $\operatorname{Tr}\left(H_{\widetilde{\gamma} \gamma^{*}}\right)=\operatorname{Tr}\left(H_{\gamma^{*}} \gamma^{*}\right)-\lambda_{k}\left(H_{\widetilde{\gamma}} \phi_{k^{*}}, \phi_{k^{*}}\right)<\operatorname{Tr}\left(H_{\gamma^{*}} \widetilde{\gamma}\right)$, which contradicts (88).

Now, assume by contradiction that $\operatorname{Tr}(\widetilde{\gamma})<N$. We distinguish two cases:

Case 1: $\widetilde{\gamma}$ is not finite rank.

In this case, we have a stronger result than the one proved in Proposition II.16:

Proposition II.17. There exists $k^{*}$ such that $0<\lambda_{k^{*}}<1$ and $\left(H_{\widetilde{\gamma}} \phi_{k^{*}}, \phi_{k^{*}}\right)<0$.

Proof. Define the set $\mathcal{J}=\left\{j: \lambda_{j}=1\right\}$. Clearly, $\mathcal{J}$ is a finite set for otherwise $\sum_{k=1}^{+\infty} \lambda_{k}=\operatorname{Tr}(\widetilde{\gamma})$ would not converge. Let the cardinality of $\mathcal{J}$ be $\bar{n}-1$ and its elements be reordered as $\mathcal{J}=\left\{\lambda_{1}, \cdots, \lambda_{\bar{n}-1}\right\}$. Since $\widetilde{\gamma}$ is not finite rank and $\mathcal{J}$ is finite, there is at least one index $j^{*}$ in its decomposition such that $\lambda_{j^{*}}<1$. Assume, by contradiction, that for every $0<\lambda_{j^{*}}<1,\left(H_{\widetilde{\gamma}} \phi_{j^{*}}, \phi_{j^{*}}\right)=0$.

By Lemma II.15, $H_{\widetilde{\gamma}}$ has infinitely many negative eigenvalues. Hence, by the min-max principle we obtain that:

$$
\begin{aligned}
& 0>\mu_{\bar{n}}\left(H_{\widetilde{\gamma}}\right)=\sup _{\nu_{1}, \ldots, \nu_{\bar{n}-1}} \inf _{\psi \in \mathcal{D}\left(H_{\widetilde{\gamma}}\right),\|\psi\|_{L^{2}}=1}\left(\psi, H_{\widetilde{\gamma}} \psi\right) \\
& \psi \in\left[\nu_{1}, \ldots, \nu_{\bar{n}-1}\right]^{\perp} \\
& \geq \inf _{\psi \in \mathcal{D}\left(H_{\widetilde{\gamma}}\right),\|\psi\|_{L^{2}}=1}\left(\psi, H_{\widetilde{\gamma}} \psi\right), \\
& \psi \in\left[\phi_{1}, \ldots, \phi_{\bar{n}-1}\right]^{\perp}
\end{aligned}
$$

where $\phi_{1}, \ldots, \phi_{\bar{n}-1}$ are the eigenfunctions of $\widetilde{\gamma}$ associated with the eigenvalues $\lambda_{1}, \cdots, \lambda_{\bar{n}-1}$ in $\mathcal{J}$. It follows that there exists $\psi_{0} \in \mathcal{D}\left(H_{\widetilde{\gamma}}\right)$ such that $\left\|\psi_{0}\right\|_{L^{2}}=1, \psi_{0} \in$ 
Hartree-Fock Theory with a Self-Generated Magnetic Field

$\left[\phi_{1}, \ldots, \phi_{\bar{n}-1}\right]^{\perp}$ and $\left(\psi_{0}, H_{\widetilde{\gamma}} \psi_{0}\right)<0$.

Define $\gamma^{*}=\sum_{k=1, k \in \mathcal{J}}^{+\infty} \phi_{k} \otimes \phi_{k}+\delta \psi_{0} \otimes \psi_{0}$, where $\delta$ is chosen in such a way that $0<\delta \leq 1$ and $\operatorname{Tr}\left(\gamma^{*}\right)=\bar{n}-1+\delta \leq N$ (note that it is always possible to choose such a $\delta$ since, given that $\widetilde{\gamma}$ is not finite rank and $\operatorname{Tr}(\widetilde{\gamma}) \leq N$, then $\bar{n}-1<N)$. We thus obtain that $\operatorname{Tr}\left(H_{\widetilde{\gamma}} \gamma^{*}\right)=\operatorname{Tr}\left(H_{\widetilde{\gamma}} \widetilde{\gamma}\right)+\delta\left(\psi_{0}, H_{\widetilde{\gamma}} \psi_{0}\right)<\operatorname{Tr}\left(H_{\widetilde{\gamma}} \widetilde{\gamma}\right)$, which is a contradiction to (88). The proof is thus concluded.

Now, choose $0<\delta<1$ such that $\lambda_{k^{*}}+\delta \leq 1$ and $\operatorname{Tr}(\widetilde{\gamma})+\delta \leq N$ (again it is always possible to find such a $\delta$ since $\lambda_{k^{*}}<1$ and we are assuming $\left.\operatorname{Tr}(\widetilde{\gamma})<N\right)$. Define $\gamma^{*}=\widetilde{\gamma}+\delta \phi_{k^{*}} \otimes \phi_{k^{*}}$. Clearly, $\operatorname{Tr}\left(\gamma^{*}\right)=\operatorname{Tr}(\widetilde{\gamma})+\delta \leq N$ and $\operatorname{Tr}\left(H_{\widetilde{\gamma} \gamma^{*}}\right)=\operatorname{Tr}\left(H_{\widetilde{\gamma}} \widetilde{\gamma}\right)+$ $\delta\left(H_{\widetilde{\gamma}} \phi_{k^{*}}, \phi_{k^{*}}\right)<\operatorname{Tr}\left(H_{\widetilde{\gamma}} \widetilde{\gamma}\right)$, which contradicts (88). Therefore, the assumption $\operatorname{Tr}(\widetilde{\gamma})<$ $N$ cannot hold if $\widetilde{\gamma}$ is not finite rank.

Case 2: $\widetilde{\gamma}$ is finite rank. In this case, $\widetilde{\gamma}$ has decomposition:

$$
\widetilde{\gamma}=\sum_{k=1}^{K_{\widetilde{\gamma}}} \lambda_{k} \phi_{k} \otimes \phi_{k}
$$

As before, from Lemma II.15 and the min-max principle, we deduce that there exists $\psi_{0} \in \mathcal{D}\left(H_{\widetilde{\gamma}}\right)$ such that $\left\|\psi_{0}\right\|_{L^{2}}=1, \psi_{0} \in\left[\phi_{1}, \ldots, \phi_{K_{\tilde{\gamma}}}\right]^{\perp}$ and $\left(\psi_{0}, H_{\widetilde{\gamma}} \psi_{0}\right)<0$. Again, choose $0<\delta<1$ such that $\operatorname{Tr}(\widetilde{\gamma})+\delta \leq N$ and define $\gamma^{*}=\widetilde{\gamma}+\delta \psi_{0} \otimes \psi_{0}$. Clearly, $\operatorname{Tr}\left(\gamma^{*}\right)=\operatorname{Tr}(\widetilde{\gamma})+\delta \leq N$ and $\operatorname{Tr}\left(H_{\widetilde{\gamma}} \gamma^{*}\right)=\operatorname{Tr}\left(H_{\widetilde{\gamma}} \widetilde{\gamma}\right)+\delta\left(\psi_{0}, H_{\widetilde{\gamma}} \psi_{0}\right)<\operatorname{Tr}\left(H_{\widetilde{\gamma}} \widetilde{\gamma}\right)$, which contradicts (88). Hence, we conclude that the assumption $\operatorname{Tr}(\widetilde{\gamma})<N$ cannot hold either when $\widetilde{\gamma}$ is finite rank.

In conclusion, we reached a contradiction in both cases, which means that the assumption $\operatorname{Tr}(\widetilde{\gamma})<N$ is never true. We thus proved that $\operatorname{Tr}(\widetilde{\gamma})=N$, which implies that $(\widetilde{\gamma}, \boldsymbol{\alpha})$ is a minimizer for (15). This concludes the proof of Theorem I.1.

Remark II.18. As it concerns Theorem I.3, we note that Lemma II.1 is still valid in the spinless case. Moreover, Lemma II.5 implies that, given a minimizing sequence $\left(\gamma_{n}, \mathbf{A}_{n}\right) \in \mathcal{C}$ for (16), we have the bound:

$$
\mathcal{E}_{\mathrm{MHF}}\left(\gamma_{n}, \mathbf{A}_{n}\right) \geq C_{1} \frac{1}{2} \operatorname{Tr}\left(\left(i \nabla-\mathbf{A}_{n}\right) \gamma_{n}\left(i \nabla-\mathbf{A}_{n}\right)\right)+\int_{\mathbb{R}^{3}}\left|\nabla \times \mathbf{A}_{n}\right|^{2}-C_{2},
$$

where $C_{1}, C_{2}$ are positive constants. Thus, $\left\{\gamma_{n}\right\}_{n \in \mathbb{N}},\left\{\nabla \times \mathbf{A}_{n}\right\}_{n \in \mathbb{N}}$ and $\left\{\mathbf{A}_{n}\right\}_{n \in \mathbb{N}}$ are uniformly bounded in $\mathcal{B}, L^{2}\left(\mathbb{R}^{3}\right)$ and $L^{6}\left(\mathbb{R}^{3}\right)$, respectively. The rest of the proof then follows as in 
Hartree-Fock Theory with a Self-Generated Magnetic Field

the spin-polarized case. Note that as a consequence of the diamagnetic and Lieb-Thirring inequalities, the kinetic energy is enough to control the potential energy for any value of $Z$. Therefore we do not need a bound of the type $Z \alpha^{2} \leq C$ in this case. We only requite $N \leq Z$, which ensures that no electron charge escapes to infinity.

\section{PROOF OF LEMMA II.15}

In the absence of magnetic fields, this lemma was proved by Lions in Ref. 10. Here we extend the result to magnetic potentials in the previously specified class. Our proof uses a similar technique to the one of Theorem XIII.6 in Ref. 31.

We first note that $\sigma_{e s s}\left(H_{1}\right) \subset[0,+\infty)$. A proof of this can be found in Appendix A of Ref. 32. Choose a function $\psi$ with the following characteristics: smooth and with compact support $\left(\psi=\left(\psi^{+}, \psi^{-}\right)^{T} \in \mathcal{C}_{0}^{\infty}\left(\mathbb{R}^{3}, \mathbb{C}^{2}\right)\right)$, radially symmetric $(\psi(\mathbf{x})=\psi(|\mathbf{x}|))$ and whose support satisfies $\operatorname{supp}(\psi)=\{1<|\mathbf{x}|<2\}$. Rescale the function in the following way: for every $\lambda>0$, define $\psi_{\lambda}=\lambda^{-3 / 2} \psi(\mathbf{x} / \lambda)$. It is clear that $\operatorname{supp}\left(\psi_{\lambda}\right)=\{\lambda<|\mathbf{x}|<2 \lambda\}$. Then:

$$
\begin{aligned}
\left(H_{1} \psi_{\lambda}, \psi_{\lambda}\right)_{L^{2}\left(\mathbb{R}^{3}, \mathbb{C}^{2}\right)}=\frac{1}{2} \int_{\mathbb{R}^{3}}\left|\nabla \psi_{\lambda}\right|_{\mathbb{C}^{2}}^{2} & +\frac{1}{2} \int_{\mathbb{R}^{3}}|\boldsymbol{\alpha}|^{2}\left|\psi_{\lambda}\right|_{\mathbb{C}^{2}}^{2}+\int_{\mathbb{R}^{3}} \mathbf{j}_{\psi}^{\lambda} \cdot \boldsymbol{\alpha}-\frac{1}{2} \int_{\mathbb{R}^{3}} \mathbf{m}_{\psi}^{\lambda} \cdot(\nabla \times \boldsymbol{\alpha}) \\
& +\int_{\mathbb{R}^{3}} v\left|\psi_{\lambda}\right|_{\mathbb{C}^{2}}^{2}+\int_{\mathbb{R}^{3}}\left(\mu * \frac{1}{|\mathbf{x}|}\right)\left|\psi_{\lambda}\right|_{\mathbb{C}^{2}}^{2},
\end{aligned}
$$

where

$$
\mathbf{j}_{\psi}^{\lambda}(\mathbf{x})=\frac{1}{2 i} \operatorname{Tr}_{\mathbb{C}^{2}}\left(\overline{\psi_{\lambda}} \cdot \nabla \psi_{\lambda}-\psi_{\lambda} \cdot \nabla \overline{\psi_{\lambda}}\right) \quad \text { and } \quad \mathbf{m}_{\psi}^{\lambda}=\left(\psi_{\lambda}, \sigma \cdot \psi_{\lambda}\right)_{\mathbb{C}^{2}}, \quad \sigma=\left(\sigma_{x}, \sigma_{y}, \sigma_{z}\right) .
$$

We want to consider (91) term by term to establish its asymptotic order when $\lambda \rightarrow+\infty$. In this way, we obtain the following:

- $\frac{1}{2} \int_{\mathbb{R}^{3}}\left|\nabla \psi_{\lambda}\right|_{\mathbb{C}^{2}}^{2}=(1 / \lambda)^{2}\left(\frac{1}{2} \int_{\mathbb{R}^{3}}|\nabla \psi|_{\mathbb{C}^{2}}^{2}\right)=o(1 / \lambda)$

- $\frac{1}{2} \int_{\mathbb{R}^{3}}|\boldsymbol{\alpha}|^{2}\left|\psi_{\lambda}\right|_{\mathbb{C}^{2}}^{2} \leq\left(\frac{1}{2 \lambda}\right) \underbrace{\left(\int_{\operatorname{supp}\left(\psi_{\lambda}\right)}|\boldsymbol{\alpha}|^{6}\right)^{1 / 3}}_{\substack{\downarrow \\ 0}}\left(\int_{\mathbb{R}^{3}}|\psi|_{\mathbb{C}^{2}}^{3}\right)^{2 / 3}=o(1 / \lambda)$, where the last equality follows from the fact that $\boldsymbol{\alpha} \in L^{6}\left(\mathbb{R}^{3}, \mathbb{R}^{3}\right)$ and $\operatorname{supp}\left(\psi_{\lambda}\right)$ is contained outside any compact set if $\lambda$ is big enough.

$$
\bullet\left|\int_{\mathbb{R}^{3}} \mathbf{j}_{\psi}^{\lambda} \cdot \boldsymbol{\alpha}\right| \leq \frac{\lambda^{-3 / 2}}{2} \underbrace{\left(\int_{\operatorname{supp}\left(\psi_{\lambda}\right)}|\boldsymbol{\alpha}|^{6}\right)^{1 / 6}}_{\substack{\downarrow \\ 0}}\left(\int_{\mathbb{R}^{3}}\left|\operatorname{Tr}_{\mathbb{C}^{2}}(\bar{\psi} \nabla \psi-\psi \nabla \bar{\psi})\right|^{6 / 5}\right)^{5 / 6}=o(1 / \lambda),
$$


Hartree-Fock Theory with a Self-Generated Magnetic Field

$$
\begin{gathered}
\cdot \begin{aligned}
\left|\frac{1}{2} \int_{\mathbb{R}^{3}} \mathbf{m}_{\Psi}^{\lambda} \cdot(\nabla \times \boldsymbol{\alpha})\right|=\left|\frac{1}{2} \int_{\mathbb{R}^{3}} \mathbf{m}_{\psi}(\mathbf{x})(\nabla \times \boldsymbol{\alpha})(\lambda \mathbf{x})\right| & \leq \frac{1}{2}\left\|\mathbf{m}_{\psi}\right\|_{L^{2}}\left(\int_{\mathbb{R}^{3}}|(\nabla \times \boldsymbol{\alpha})(\lambda \mathbf{x})|^{2}\right)^{1 / 2} \\
& =\frac{1}{2}\|\psi\|_{L^{4}}^{2} \lambda^{-3 / 2}\|\nabla \times \boldsymbol{\alpha}\|_{L^{2}}=o(1 / \lambda),
\end{aligned} \\
\qquad \int_{\mathbb{R}^{3}} v\left|\psi_{\lambda}\right|_{\mathbb{C}^{2}}^{2}=\int_{\operatorname{supp}(\psi)} v(\lambda \mathbf{x})|\psi(\mathbf{x})|_{\mathbb{C}^{2}}^{2} \\
=-\frac{1}{\lambda} Z\left(\int_{\operatorname{supp}(\psi)} \frac{|\psi(\mathbf{x})|_{\mathbb{C}^{2}}^{2}}{|\mathbf{x}|}\right)+o(1 / \lambda),
\end{gathered}
$$

since

$$
\begin{aligned}
v(\lambda \mathbf{x})=-\sum_{k=1}^{K} & \frac{Z_{k}}{\left|\lambda \mathbf{x}-\mathbf{R}_{k}\right|}=-\frac{1}{\lambda} \sum_{k=1}^{K} \frac{Z_{k}}{\left|\mathbf{x}-\frac{\mathbf{R}_{k}}{\lambda}\right|} \stackrel{\operatorname{supp}(\psi)}{=}-\frac{1}{\lambda} \frac{Z}{|\mathbf{x}|}+o(1 / \lambda) . \\
-\int_{\mathbb{R}^{3}}\left(\mu * \frac{1}{|\mathbf{x}|}\right)\left|\psi_{\lambda}\right|_{\mathbb{C}^{2}}^{2} & =\frac{1}{\lambda} \int_{\mathbb{R}^{3}}\left(\mu_{\lambda} * \frac{1}{|\mathbf{x}|}\right)|\psi|_{\mathbb{C}^{2}}^{2} \quad \mu_{\lambda}=\lambda^{3} \mu(\lambda \cdot) \\
& =\frac{1}{\lambda} \int_{\mathbb{R}^{3}}\left(|\psi|_{\mathbb{C}^{2}}^{2} * \frac{1}{|\mathbf{x}|}\right) d \mu_{\lambda} \\
& =\frac{1}{\lambda} \iint_{\mathbb{R}^{3} \times \mathbb{R}^{3}}|\psi(\mathbf{y})|_{\mathbb{C}^{2}}^{2} \max (|\mathbf{x}|,|\mathbf{y}|)^{-1} d \mu_{\lambda}(\mathbf{x}) d \mathbf{y} \\
& \leq \frac{1}{\lambda} \mu\left(\mathbb{R}^{3}\right) \int_{\mathbb{R}^{3}} \frac{|\psi(\mathbf{y})|_{\mathbb{C}^{2}}^{2}}{|\mathbf{y}|} d \mathbf{y}
\end{aligned}
$$

Now, since by hypothesis $\left(\mu\left(\mathbb{R}^{3}\right)-Z\right)<0$, it is clear that if we choose $\lambda$ big enough (say $\lambda>Q$ ), we have that

$$
\left(H_{1} \psi_{\lambda}, \psi_{\lambda}\right)_{L^{2}\left(\mathbb{R}^{3}, \mathbb{C}^{2}\right)}<0 .
$$

For any $n \in \mathbb{N}$, define $\psi_{n}=\psi_{2^{n} Q}$. Note that $\left\{\psi_{n}\right\}_{n}$ have disjoint supports, are orthonormal, satisfy $\left(\psi_{n}, H_{1} \psi_{n}\right)_{L^{2}\left(\mathbb{R}^{3}, \mathbb{C}^{2}\right)}<0$ and $\left(\psi_{n}, H_{1} \psi_{m}\right)_{L^{2}\left(\mathbb{R}^{3}, \mathbb{C}^{2}\right)}=0$ if $n \neq m$. For any $n \in \mathbb{N}$, define $V_{n}=\operatorname{span}\left\{\psi_{1}, \cdots, \psi_{n}\right\}$. Note that, $\left.P_{n} H_{1} P_{n}\right|_{V_{n}}$ has eigenvalues $\left\{\left(\psi_{m}, H_{1} \psi_{m}\right)_{L^{2}\left(\mathbb{R}^{3}, \mathbb{C}^{2}\right)}\right\}_{m=1}^{n}$. By the Raleigh-Ritz principle (see Theorem XIII.3 in Ref. 31) we obtain that

$$
\mu_{n}\left(H_{1}\right) \leq \sup _{1 \leq m \leq n}\left\{\left(\psi_{m}, H_{1} \psi_{m}\right)_{L^{2}\left(\mathbb{R}^{3}, \mathbb{C}^{2}\right)}\right\}<0 .
$$

Since $\sigma_{\text {ess }}\left(H_{1}\right) \subset[0,+\infty)$, and $n$ was arbitrary, this means that $H_{1}$ has infinitely many negative eigenvalues.

\section{ACKNOWLEDGEMENTS}

The work of CJGC and SC was supported by the National Science Foundation (NSF) via Grant DMS No. 1065942. CJGC acknowledges support from NSF Grant DMS 0645766 and the Bizkaia Talent program through the Basque Center for Applied Mathematics (BCAM). 
Hartree-Fock Theory with a Self-Generated Magnetic Field

\section{REFERENCES}

${ }^{1}$ J. Fröhlich, E. H. Lieb, and M. Loss, Comm. Math. Phys. 104, 251 (1986).

${ }^{2}$ E. H. Lieb and M. Loss, Comm. Math. Phys. 104, 271 (1986).

${ }^{3}$ M. Loss and H.-T. Yau, Comm. Math. Phys. 104, 283 (1986).

${ }^{4}$ E. H. Lieb, M. Loss, and J. P. Solovej, Phys. Rev. Lett. 75, 985 (1995).

${ }^{5}$ E. Lieb and W. Thirring, Phys. Rev. Lett. 35, 687 (1975).

${ }^{6}$ E. H. Lieb and M. Loss, Analysis, 2nd ed., Graduate Studies in Mathematics, Vol. 14 (American Mathematical Society, Providence, RI, 2001) pp. xxii+346.

${ }^{7}$ E. H. Lieb, H. Siedentop, and J. P. Solovej, J. Statist. Phys. 89, 37 (1997), dedicated to Bernard Jancovici.

${ }^{8}$ L. Bugliaro, C. Fefferman, J. Fröhlich, G. M. Graf, and J. Stubbe, Comm. Math. Phys. 187, 567 (1997).

${ }^{9}$ E. H. Lieb and B. Simon, Comm. Math. Phys. 53, 185 (1977).

${ }^{10}$ P.-L. Lions, Comm. Math. Phys. 109, 33 (1987).

${ }^{11}$ G. Friesecke, Archive for Rational Mechanics and Analysis 169, 35 (2003).

${ }^{12}$ M. Lewin, Journal of Functional Analysis 260, 3535 (2011).

${ }^{13}$ M. Enstedt and M. Melgaard, Nonlinear Anal. 69, 2125 (2008).

${ }^{14}$ M. J. Esteban and P.-L. Lions, in Partial differential equations and the calculus of variations, Vol. I, Progr. Nonlinear Differential Equations Appl., Vol. 1 (Birkhäuser Boston, Boston, MA, 1989) pp. 401-449.

${ }^{15}$ E. H. Lieb, Phys. Rev. A 29, 3018 (1984).

${ }^{16}$ J. P. Solovej, Ann. of Math. (2) 158, 509 (2003).

${ }^{17}$ E. H. Lieb and B. Simon, Advances in Math. 23, 22 (1977).

${ }^{18}$ L. Erdős and J. P. Solovej, Communications in Mathematical Physics 294, 229 (2009).

${ }^{19}$ W. Kohn and L. J. Sham, Phys. Rev. (2) 140, A1133 (1965).

${ }^{20}$ D. Gontier, Nonlinearity 28, 57 (2015).

${ }^{21}$ A. Anantharaman and E. Cancès, Ann. Inst. H. Poincaré Anal. Non Linéaire 26, 2425 (2009).

${ }^{22}$ T. L. Gilbert, Phys. Rev. B 12, 2111 (1975).

${ }^{23}$ S. M. Valone, J. Chem. Phys. 73, 1344 (1980). 
Hartree-Fock Theory with a Self-Generated Magnetic Field

${ }^{24}$ I. Catto, C. Le Bris, and P.-L. Lions, Ann. Inst. H. Poincaré Anal. Non Linéaire 18, 687 (2001).

${ }^{25}$ J. P. Solovej, Invent. Math. 104, 291 (1991).

${ }^{26}$ A. J. Coleman, Rev. Modern Phys. 35, 668 (1963).

${ }^{27}$ M. Reed and B. Simon, Methods of modern mathematical physics. I, 2nd ed. (Academic Press, Inc. [Harcourt Brace Jovanovich, Publishers], New York, 1980) pp. xv+400, functional analysis.

${ }^{28}$ E. H. Lieb, Phys. Rev. Lett. 46, 457 (1981).

${ }^{29}$ V. Bach, Comm. Math. Phys. 147, 527 (1992).

${ }^{30} \mathrm{M}$. Reed and B. Simon, Methods of modern mathematical physics. II. Fourier analysis, selfadjointness (Academic Press [Harcourt Brace Jovanovich, Publishers], New York-London, 1975) pp. $\mathrm{xv}+361$.

${ }^{31} \mathrm{M}$. Reed and B. Simon, Methods of modern mathematical physics. IV. Analysis of operators (Academic Press [Harcourt Brace Jovanovich, Publishers], New York-London, 1978) pp. $\mathrm{xv}+396$.

${ }^{32}$ L. Erdős, Comm. Math. Phys. 170, 629 (1995). 